\title{
Interpolation of multidimensional diabatic potential energy matrices
}

\author{
Oded Godsi \\ Research School of Chemistry, Australian National University, Canberra ACT 0200, Australia \\ Christian R. Evenhuis \\ Research School of Chemistry, Australian National University, Canberra ACT 0200, Australia \\ and Theoretische Chemie, Fakultät für Chemie, Universität Bielefeld, Universitätsstrasse 25, \\ 33615 Bielefeld, Germany \\ Michael A. Collins ${ }^{\text {a) }}$ \\ Research School of Chemistry, Australian National University, Canberra ACT 0200, Australia
}

(Received 30 May 2006; accepted 31 July 2006; published online 11 September 2006)

\begin{abstract}
A method for constructing diabatic potential energy matrices by interpolation of ab initio quantum chemistry data is described and tested. This approach is applicable to any number of interacting electronic states, and relies on a formalism and a computational procedure that are more general than those presented previously for the case of two electronic states. The method is tested against an analytic model for three interacting electronic states of $\mathrm{NH}_{3}^{+}$. (C) 2006 American Institute of Physics. [DOI: 10.1063/1.2338912]
\end{abstract}

\section{INTRODUCTION}

Much of theoretical chemical reaction dynamics have been pursued within the Born-Oppenheimer approximation. ${ }^{1}$ In this adiabatic approximation, the "slow" motion of the atomic nuclei is separated from that of the "fast" electronic degrees of freedom. The electronic Schrödinger equation, which is parametrically dependent on the nuclear coordinates, can be solved by modern $a b$ initio quantum chemistry methods to give the electronic energy at a given nuclear configuration. The resultant potential energy surface (PES), for a molecule of $N$ atoms, is the $3 N-6$ dimensional function of nuclear configuration that describes the potential energy governing the motion of the atomic nuclei. For reactions involving more than three atoms, it is not feasible for a highly accurate $a b$ initio calculation of the potential energy to be performed at every relevant molecular configuration. It is necessary to approximate the PES. Modified Shepared interpolation of $a b$ initio data has been shown to provide a satisfactory approach to PES approximation for reactions involving several atoms. ${ }^{2}$

However, the Born-Oppenheimer approximation is not always sufficient. Many important chemical processes involve multiple electronic states and nondiabatic dynamics of the atomic nuclei. Nevertheless, the adiabatic electronic basis (the basis in which the electronic Hamiltonian is diagonal) can be used to evaluate the quantities needed for the nonadiabatic dynamics. The nuclear wave functions for different electronic states are coupled by so-called derivative coupling terms. From perturbation theory or exact calculation, ${ }^{3}$ it can be shown that, near a degeneracy, the derivative coupling between two electronic states is inversely proportional to the difference of their electronic energies. This derivative coupling can be neglected if the energies of the electronic states are well separated. However, as the nuclear coordinates ap-

${ }^{a)}$ Electronic mail: collins@rsc.anu.edu.a proach a point where an electronic degeneracy occurs, the derivative coupling becomes singular, ${ }^{3}$ which causes the nuclear wave functions for different electronic states to be strongly coupled. Nuclear dynamics in the adiabatic basis is difficult to implement due to this singularity in the derivative coupling. In addition, the second order Taylor series used in modified Shepard interpolation cannot describe the characteristic conical shape of the potential energy surfaces near such a degeneracy, nor the singularity in the coupling terms. Hence, it would be difficult to construct such surfaces by interpolation in the manner used for adiabatic PES. These difficulties are removed by a transformation to a diabatic basis.

Near a degeneracy (and to first order in the displacements of nuclear coordinates) it is possible to transform the electronic basis so that the derivative couplings are removed (to first order) and the diagonal adiabatic PESs are replaced by a matrix of potential energies that is a smooth function of nuclear displacements. ${ }^{4}$ This representation is referred to as a diabatic basis. Nuclear dynamics can be more easily performed in this electronic basis, and modified Shepard interpolation can be applied to approximate the elements of the diabatic potential energy matrix (DPEM). In two previous papers, we presented an automated method for the construction and interpolation of diabatic potential energy matrices, for the special case where just two electronic states were considered. ${ }^{5}$ This approach was successfully tested by comparison with the model analytic diabatic surfaces of Varandas et al. ${ }^{6}$ for $\mathrm{H}_{3}$ and for ab initio calculation of a diabatic potential matrix for the two lowest states of $\mathrm{H}_{3}$.

However, the general case where many (nstates) electronic states may be degenerate (either pairwise or with higher degeneracy) is not described by this earlier approach. In general, the adiabatic to diabatic transformation (ADT) is represented by an nstates $\times$ nstates unitary matrix and the nuclear diabatic dynamics is governed by an nstates 
$\times$ nstates Hermitian potential energy matrix. For the nonrelativistic Hamiltonian, the ADT transformation is represented by an orthogonal matrix and the diabatic potential energy matrix is real and symmetric. The automated construction of such interpolated real diabatic potential matrices is the subject of this paper. The general case presents additional technical problems to those encountered in the two state case. Where possible, the detailed descriptions of the algorithms devised to treat these problems have been included in the appendices. To test the methods presented here, we have again employed an analytic model for comparison. ${ }^{7}$

Although multiple electronic states are considered to be coupled in the treatment present herein, inevitably many other electronic states are ignored. The finite basis of electronic states which we use must always be incomplete. Because of the existence of other electronic states, the derivative coupling between any two (or a finite number of) states cannot be totally removed by the ADT. An exact ADT (that is, one which completely removes the derivative coupling) is not possible. However, an approximate (nonunique) orthogonal transformation of the electronic states which removes the singularity in the derivative coupling between the transformed states can be found. ${ }^{8}$ If, after such an approximate ADT, the residual derivative coupling can be ignored within the Born-Oppenheimer approximation, the dynamics performed in this quasidiabatic basis should be nearly equivalent to dynamics performed in the adiabatic basis. It is important to point out that the test case we consider in this first exposition of the general nstates approach does not examine the effect of such residual couplings, which are absent in the analytic model. $^{7}$

The paper is set out as follows. The established formalism for the adiabatic to diabatic transformation is presented very briefly in Sec. II. The method for constructing the diabatic potential energy matrix by interpolation is presented in Sec. III. Several important issues are discussed therein, while many of the technical details are presented in the appendices. Section IV describes the automated procedure used to accumulate the data employed in the interpolation, concentrating on aspects which are specific to the DPEM case as opposed to a single adiabatic PES. To test this whole approach, we have constructed an interpolated DPEM which approximates an analytic form for a DPEM for $\mathrm{NH}_{3}^{+}$. In Sec. V, results are presented for surface hopping dynamics derived from the interpolated DPEM and the original analytic form. Some brief concluding remarks are presented in the last section.

\section{BACKGROUND THEORY}

The nonrelativistic, spin-free, Hamiltonian for a molecule can be written as

$$
H=-\frac{1}{2 M} \nabla^{2}+H_{e}
$$

where $H_{e}$ is the usual electronic Hamiltonian (which is parametrically dependent on the positions of the nuclei) in a.u. in which the unit of length is the Bohr, the unit of mass is the electron mass, the nuclear coordinates are mass scaled Cartesians, and $M$ represents the ratio of amu to the electron mass. In the adiabatic Born-Oppenheimer approximation, the eigenfunctions of $H_{e}$ are evaluated:

$$
H_{e} \psi_{n}(\mathbf{x} ; \mathbf{X})=E_{n}(\mathbf{X}) \psi_{n}(\mathbf{x} ; \mathbf{X}),
$$

where $\mathbf{x}$ and $\mathbf{X}$ represent the electronic and nuclear coordinates, respectively, and $E_{n}(\mathbf{X})$ is the usual adiabatic PES for the $n$th electronic state. The total molecular wave function is expanded in this so-called adiabatic basis [using a finite number of states (nstates)] as

$$
\Psi(\mathbf{x} ; \mathbf{X})=\sum_{n=1}^{\text {nstates }} \chi_{n}(\mathbf{X}) \psi_{n}(\mathbf{x} ; \mathbf{X})=\underline{\chi}^{T}(\mathbf{X}) \underline{\psi}(\mathbf{x} ; \mathbf{X}) .
$$

Substituting this expansion into the Schrödinger equation, and using the orthogonality of the electronic wave functions, a set of coupled equations for the nuclear wave functions is obtained:

$$
-\frac{1}{2 M}\left[\nabla^{2}+2 \underline{\underline{\mathbf{F}}}+\underline{\underline{G}}\right] \underline{\underline{\chi}}+\underline{\underline{E}} \underline{\underline{\chi}}=E \underline{\underline{\chi}},
$$

where $\underline{\underline{E}}$ is the diagonal matrix of potential energies and $\underline{\underline{\mathbf{F}}}$ and $\underline{\underline{G}}$ are matrices of derivative couplings:

$$
\begin{aligned}
& \mathbf{F}_{n, m}=\int \psi_{n}(\mathbf{x} ; \mathbf{X}) \nabla \psi_{m}(\mathbf{x} ; \mathbf{X}) d \mathbf{x}, \\
& G_{n, m}=\int \psi_{n}(\mathbf{x} ; \mathbf{X}) \nabla^{2} \psi_{m}(\mathbf{x} ; \mathbf{X}) d \mathbf{x} .
\end{aligned}
$$

Note that $\underline{\underline{\mathbf{F}}}$ is a matrix with elements given by $3 N$ dimensional Cartesian vectors. If we neglect the coupling of the nstates (electronic states) to all other states, then $\underline{\underline{G}}$ can be approximated as

$$
\underline{\underline{G}} \approx \nabla \cdot \underline{\underline{\mathbf{F}}}+\underline{\underline{\mathbf{F}}} \cdot \underline{\underline{\mathbf{F}}}
$$

Equation (2.4) is then simplified to

$$
-\frac{1}{2 M}[\nabla+\underline{\underline{\mathbf{F}}}]^{2} \underline{\underline{\chi}}+\underline{\underline{E}} \underline{\chi}=E \underline{\underline{\chi}} .
$$

To avoid the difficulties associated with performing nuclear dynamics in the adiabatic representation one can transform the adiabatic electronic wave functions to the so-called "diabatic" electronic wave functions.

There are two main approaches for obtaining the ADT; the first one focuses on reducing the changes in the electronic wave functions. ${ }^{9}$ This class of approaches includes those that use the smoothness of molecular properties such as the dipole moment. ${ }^{10}$ Those approaches that are based on the electronic wave functions may not be the preferred choice when the nuclear displacement from the conical intersection is large; in such cases, it is less likely that the ADT is well behaved and the residual couplings are small. The second approach focuses on removing the derivative coupling 
or, more accurately, on removing the singularity in the derivative coupling and minimizing the residual coupling. ${ }^{11,12}$ Although historically much research has centered on the minimization of changes in electronic wave functions, here we focus on minimizing the derivative couplings.

The ADT is an orthogonal transformation of the nstates electronic wave functions of interest, and the diabatic electronic wave functions $\tilde{\psi}(\mathbf{x}, \mathbf{X})$ are related to the adiabatic electronic wave functions $\psi(\mathbf{x}, \mathbf{X})$ by

$$
\widetilde{\psi}(\mathbf{x}, \mathbf{X})=\underline{A}^{T}(\mathbf{X}) \underline{\psi}(\mathbf{x}, \mathbf{X}),
$$

where $\underset{\equiv}{A}(\mathbf{X})$ is the ADT matrix. In order for the total molecular wave function of Eq. (2.3) to remain unchanged by the transformation, the corresponding diabatic nuclear wave functions $\tilde{\chi}(\mathbf{X})$ are related to the adiabatic nuclear wave functions by

$$
\underline{\widetilde{\chi}}(\mathbf{X})=\underline{A}^{T}(\mathbf{X}) \underline{\chi}(\mathbf{X}) \text {. }
$$

After the transformation to the diabatic basis, the Schrödinger equation (2.7) becomes

$$
\left[-\frac{1}{2 M}(\nabla+\underline{\underline{\mathbf{F}}})^{2}+\underline{D}\right] \underline{\tilde{\chi}}=E \underline{\tilde{\chi}},
$$

where the derivative coupling in this diabatic basis is

$$
\underset{\underline{\mathbf{F}}}{=} \underline{A}^{T} \nabla \underset{\underline{A}}{A}+\underline{\underline{A}}^{T} \underline{\underline{\mathbf{F}}}=\underline{\underline{A}}
$$

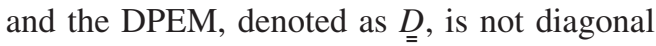

$$
\begin{aligned}
D_{n, m} & =\left\langle\widetilde{\psi}_{n}\left|H_{e}\right| \tilde{\psi}_{m}\right\rangle=\left\langle\sum_{k} A_{k, n} \psi_{k}\left|H_{e}\right| \sum_{l} A_{l, m} \psi_{l}\right\rangle \\
& =\sum_{k, l} A_{k, n}\left\langle\psi_{k}\left|H_{e}\right| \psi_{l}\right\rangle A_{l, m}=\sum_{k, l} A_{k, n} \delta_{k, l} E_{l} A_{l, m} .
\end{aligned}
$$

In matrix form,

$$
\underset{\underline{D}}{=} \underline{\underline{A}}^{T} \underline{\underline{E}} \underset{=}{A}
$$

Ideally, $\underset{\equiv}{A}$ would be chosen so that the derivative coupling is completely removed. Requiring the derivative coupling in the diabatic basis [see Eq. (2.11)] to be zero leads to

$$
\nabla \underset{=}{A}=-\underline{\underline{\mathbf{F}}} \underset{\underline{A}}{\underline{A}} .
$$

Then the diabatic nuclear Schrödinger equation is simplified to

$$
\left[-\frac{1}{2 M} \nabla^{2}+\underline{D}\right] \underline{\tilde{\chi}}=E \underline{\tilde{\chi}} .
$$

Formally, the solution to Eq. (2.14) is given by an integral along some path $\mathbf{S}(t)$ such that $\mathbf{S}(0)=\mathbf{X}_{1}$ and $\mathbf{S}(1)=\mathbf{X}_{2}$

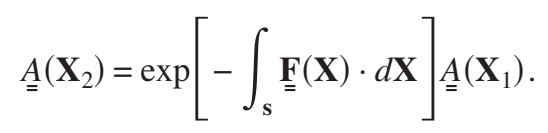

However, it has been well known for many years ${ }^{11,13}$ that there is no unique solution for $\underset{=}{A}$ in (2.14) unless nstates $=\infty$. The derivative coupling in the infinite diabatic basis is exactly zero. However, if the electronic basis set has been restricted to a finite number of states, there is no unique value for the integral in Eq. (2.16), and the ADT is not uniquely defined. Alternatively, we can consider that the ADT can only remove part of the derivative coupling, $\underset{=}{\mathbf{F}}$, between the states of interest (the so-called removable component). The general characteristics of the removable and nonremovable components have been discussed by Kendrick et $a l .{ }^{14}$ In summary, near a degeneracy only the removable coupling is singular. Degenerate perturbation theory shows that near a conical intersection the nonremovable couplings are insignificant. ${ }^{15}$ This means that an approximate solution of (2.14) can give an $\underset{\underline{\mathbf{F}}}{\widetilde{T}}$ matrix in (2.11) which is always finite. This residual coupling matrix can be ignored, both near to or far from electronic degeneracies, in dynamics calculations due the $1 / M$ prefactor in (2.10), just as one does in the usual Born-Oppenheimer approximation for energetically well-separated electronic states. Here we show how such an approximate solution of (2.14) can be obtained by interpolation of $a b$ initio data. More importantly, we use an interpolation approach to construct the diabatic potential energy matrix which is needed to study the chemical dynamics described by Eq. (2.15). In the absence of the nonremovable coupling, this interpolation approach would be exact in the limit of infinite data density. However, the presence of nonremovable couplings and the inevitably limited density of $a b$ initio data combine to ensure that the interpolation approach produces only an approximate diabatic potential energy matrix. For the simple analytic model considered below (for which nonremovable couplings are absent), we show that the interpolation is sufficiently accurate even for very limited data.

\section{DIABATIC INTERPOLATION}

The DPEM is a smooth function of the nuclear coordinates and can be expanded locally as a Taylor series. Globally, the DPEM is constructed as a weighted sum of Taylor series from a scattered set of geometries (a modified Shepard interpolation $^{16}$ ):

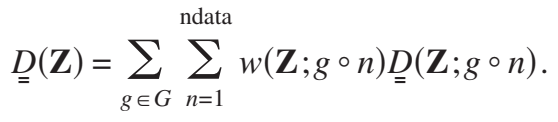

Equation (3.1) is very similar in structure to that for modified Shepard interpolation of a single PES. This approximation for a PES has been presented and discussed in several papers, ${ }^{2,17-19}$ but we will carefully define each of the terms in Eq. (3.1). The vector $\mathbf{Z}$ represents the nuclear molecular internal coordinates, corresponding to the Cartesian coordinates $\mathbf{X} . \underset{=}{D}(\mathbf{Z} ; g \circ n)$ represents a matrix in which each element is a (second order) Taylor series which approximates the corresponding element of $\underset{\underline{D}}{\boldsymbol{Z}} \mathbf{Z})$. The Taylor series is centered at a molecular configuration $g \circ \mathbf{Z}(n)$, abbreviated to $g$ ' $n$ and usually referred to as a "data point." If $\mathbf{Z}(n)$ represents some particular (numbered) data point, then $g \circ \mathbf{Z}(n)$ represents the configuration obtained by acting on $\mathbf{Z}(n)$ with an element $g$ of the molecular symmetry group $G$. In general, 
$G$ is the complete nuclear permutation inversion (CNPI) group. $w(\mathbf{Z} ; g \circ n)$ represents the weight (valued between 0 and 1) that this Taylor series has when each Taylor series is evaluated at $\mathbf{Z}$. A quantitative description of this weight is presented below, but in simple terms, the weight $w(\mathbf{Z} ; g \circ n)$ is larger as $g \circ \mathbf{Z}(n)$ is "closer" to $\mathbf{Z}$; the closer the data point is to the configuration where $\underset{=}{D}$ is evaluated, the more accurate the Taylor series should be, and the higher the weight should be.

Although Eq. (3.1) is superficially similar to the corresponding expression for an interpolated PES, there are major differences. The molecular coordinates needed to describe a DPEM are different from those needed to describe a PES. The symmetry of a DPEM (described by the action of each group element $g$ ) is different from the symmetry of a PES. The coefficients that appear in the Taylor series $\underset{\underline{D}}{(\mathbf{Z} ; g \circ n)}$ are evaluated from $a b$ initio quantum chemistry calculations, as for a PES, but require a much more complicated process to evaluate them. In the remainder of this section, each term in Eq. (3.1) is described in detail.

\section{A. Molecular coordinates}

A PES is invariant to translation, rotation, or inversion of the molecule, and so a PES can be expressed in terms of molecular coordinates which are also invariant to translation, rotation, or inversion, for example, atom-atom distances. If the nuclear coordinates of a molecule are all shifted by a simple translation, the adiabatic electronic wave functions are also simply translated. Similarly, if the nuclear coordinates are rotated, the electronic coordinates in the adiabatic electronic wave functions are rotated in the same way. The overall sign of an electronic wave function, evaluated at some nuclear configuration, $\mathbf{Z}(n)$, is arbitrary. If the electronic state is nondegenerate, then permutation or inversion of the nuclei can, at most, change the sign of the electronic wave function. ${ }^{20}$ However, if two or more electronic states are degenerate at some $\mathbf{Z}(n)$, then permutation or inversion of the nuclei can transform the degenerate wave functions into some new (unitary) combination of the original wave functions. As a result, the DPEM may be transformed in a nontrivial manner from its value at $\mathbf{Z}(n)$ to its value at $g$ $\circ \mathbf{Z}(n)$. Thus, the DPEM is invariant to translation and rotation, but not invariant to permutation or inversion. Therefore the DPEM can be expressed in terms of molecular coordinates which are invariant to translation and rotation, but which are not invariant to permutation or inversion. Previously, the modified Shepard interpolation for PES has been evaluated using atom-atom distances (actually the reciprocals of these bond lengths) as coordinates. These variables are invariant to inversion and hence not suitable, by themselves, as coordinates to describe a DPEM.

The fundamental nuclear coordinate variables which are invariant to translation and rotation but not to inversion are the "dot-cross-product" variables (see Ref. 21 and references therein). If $\mathbf{r}_{1}=\mathbf{x}_{1}-\mathbf{x}_{2}, \mathbf{r}_{2}=\mathbf{x}_{1}-\mathbf{x}_{3}$, and $\mathbf{r}_{3}=\mathbf{x}_{1}-\mathbf{x}_{4}$ represent Cartesian vectors between the positions of four atoms, then

$$
d_{i}=\mathbf{r}_{1} \cdot\left(\mathbf{r}_{2} \times \mathbf{r}_{3}\right)
$$

is invariant to translation and rotation but not to inversion. Like the atom-atom distances $\left\|\mathbf{r}_{i}\right\|$, the variables $d_{i}$ are transformed in a nontrivial, but easily evaluated, way under permutation of the nuclei. There are nbond $=N(N-1) / 2$ atomatom distances $r_{i}$ and $\operatorname{ndotc}=N ! /[(N-4) ! 4 !]$ distinct dotcross-product variables. Together these variables form a faithful representation of the CNPI group. The coordinates $\mathbf{Z}$ used hereafter are defined as

$$
\begin{aligned}
& Z_{i}=1 / r_{i} \text { for } i=1, \ldots, \text { nbond }, \\
& Z_{\text {nbond }+i}=\frac{d_{i}}{\left[\sum_{k=1}^{6} r_{k}^{(i)} / 6\right]^{4}} \text { for } i=1, \ldots, \text { ndotc }
\end{aligned}
$$

The four atoms which define the $i$ th dot-cross variable $d_{i}$ also define the six atom-atom distances which appear in the denominator of Eq. (3.3). This scale factor is introduced to retain the same dimensionality for all elements of $\mathbf{Z}$. A totally symmetric sum of bond lengths is chosen to ensure that the symmetry of these coordinates is totally determined by the symmetry of the $d_{i}$. Incorporation of both atom-atom distances and dot-cross-product variables in $\mathbf{Z}$ ensures that functions which are either even or odd under inversion can be described. The dot-cross-product variables are not defined (or necessary) for systems of less than four atoms (as considered in earlier work ${ }^{5}$ ).

Together, the nbond+ndotc elements of $\mathbf{Z}$ form a socalled redundant set of internal molecular coordinates. A set of $3 N-6$ locally independent internal coordinates can be formed from the elements of $\mathbf{Z}$ via a simple generalization of the approach used previously for PES. ${ }^{18}$ Briefly, at a data point with Cartesian coordinate $\mathbf{X}(n)$, and internal coordinates $\mathbf{Z}(n)$, a "Wilson $B$ matrix" is defined by

$$
\begin{aligned}
& B_{\alpha i}(n)=\left.\frac{\partial Z_{\alpha}}{\partial X_{i}}\right|_{\mathbf{X}=\mathbf{X}(n)}, \quad \alpha=1, \ldots, \text { nbond + ndotc, } \\
& i=1, \ldots, 3 N
\end{aligned}
$$

A singular value decomposition of $\underset{\underline{B}}{(n)}$ is given by

$$
\underline{\underline{B}}(n)=\underline{\underline{U}}(n) \underset{=}{\Lambda}(n) \underline{\underline{V}}^{T}(n) .
$$

Here $U(n)$ is an (nbond+ndotc) $\times($ nbond+ndotc) unitary matrix, and $\underline{\underline{V}}(n)$ is a $3 N \times 3 N$ unitary matrix. $\Lambda(n)$ is an (nbond + ndotc) $\times 3 N$ diagonal matrix of (positive semidefinite) singular values. Only $3 N-6$ of these singular values, $\lambda_{k}$, can be nonzero, so we order them to put these nonzero elements as the first $3 N-6$ terms on the diagonal (and order the columns of $\underline{\underline{U}}$ and $\underline{\underline{V}}$ correspondingly). The singular value decomposition then allows locally independent internal 
coordinates $\xi$ to be defined as

$$
\xi_{k}=\sum_{\alpha=1}^{\substack{\text { nbond } \\+ \text { ndotc }}} U_{k, \alpha}(n) Z_{\alpha} / \lambda_{k}, \quad k=1, \ldots, 3 N-6 .
$$

For configurations close to $\mathbf{Z}(n)$, these are well-defined and locally independent coordinates which determine the shape and handedness of the molecule.

\section{B. Taylor series}

To construct a Taylor series for the DPEM about $\mathbf{Z}(n)$,

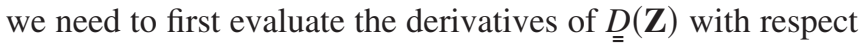
to the nuclear coordinates. From Eq. (2.13), we see that this requires the derivatives of the ADT matrix, $A$, and the adiabatic energies. The first derivative of the ADT matrix comes directly from Eq. (2.14). The second derivative is derived by reapplication of the definition of the first derivative.

$$
\begin{aligned}
& \left.\frac{\partial}{\partial X_{a}} \stackrel{A}{=}\right|_{\mathbf{X}=\mathbf{X}(n)}=-\underline{F}_{a} \mid \mathbf{X}=\mathbf{X}(n) \stackrel{A}{=}(n), \\
& \left.\frac{\partial^{2}}{\partial X_{b} \partial X_{a}} \stackrel{A}{A}\right|_{\mathbf{X}=\mathbf{X}(n)}=\left.\left(-\frac{\partial}{\partial X_{b}}=F_{a}+\underline{F}_{a} \underline{F}_{b}\right)\right|_{\mathbf{X}=\mathbf{X}(n)} \underset{=}{A}(n) .
\end{aligned}
$$

Here $\underset{=}{A}(n)$ denotes the value of the ADT matrix at $\mathbf{X}=\dot{\mathbf{X}}(n)$. As $\mathbf{F}$ is antisymmetric, the derivatives of the transpose of the ADT matrix are

$$
\begin{aligned}
& \left.\frac{\partial}{\partial X_{a}} A^{T}\right|_{\mathbf{X}=\mathbf{X}(n)}={\underset{A}{A}}^{T}(n) \underset{\underline{F}}{\underline{F}} \mid \mathbf{X}=\mathbf{X}(n), \\
& \left.\frac{\partial^{2}}{\partial X_{b} \partial X_{a}} A^{T}\right|_{\mathbf{X}=\mathbf{X}(n)}=\left.\underline{A}_{=}^{T}(n)\left(\frac{\partial}{\partial X_{b}} F_{a}+\underline{F}_{b} \underline{F}_{a}\right)\right|_{\mathbf{X}=\mathbf{X}(n)} .
\end{aligned}
$$

The derivatives of the diabatic potential are then

$$
\begin{aligned}
& \left.\underline{\underline{D}}\right|_{\mathbf{X}=\mathbf{X}(n)}=\left.\underline{\underline{A}}^{T}(n) \underline{\underline{E}}\right|_{\mathbf{X}=\mathbf{X}(n)} \stackrel{A}{A}_{(n)}, \\
& \left.\frac{\partial}{\partial X_{a}} \underline{D}\right|_{\mathbf{X}=\mathbf{X}(n)}=\left.\underline{A}^{T}(n)\left(\frac{\partial}{\partial X_{a}} \underline{\underline{E}}+[\underline{\underline{F}} a, \underline{\underline{E}}]_{-}\right)\right|_{\mathbf{X}=\mathbf{X}(n)} \underset{=}{A}(n), \\
& \frac{\partial^{2}}{\partial X_{b} \partial X_{a}}=\left.\right|_{\mathbf{X}=\mathbf{X}(n)}=\underline{A}_{=}^{T}(n)\left(\left[\frac{\partial}{\partial X_{b}} \underline{F_{a}}, \underline{\underline{E}}\right]_{-}+\left[\underline{\underline{F}}_{b} \underline{F}_{a}, E\right]_{+}\right. \\
& +\left[\underline{F}_{a}, \frac{\partial}{\partial X_{b}} \underline{\underline{E}}\right]_{-}+\left[\underline{\underline{F}}, \frac{\partial}{\partial X_{a}} \underline{\underline{E}}\right]_{-} \\
& -\underline{\underline{F}}_{a} \underline{\underline{E}}_{\underline{\underline{F}}} b-\underline{\underline{F}}_{b} \underline{\underline{E}} \underline{\underline{F}}_{a} \\
& \left.+\frac{\partial^{2}}{\partial X_{b} \partial X_{a}} \underline{=}\right)\left.\right|_{\mathbf{X}=\mathbf{X}(n)} \underset{=}{A}(n),
\end{aligned}
$$

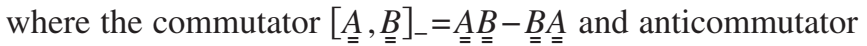
$[\underset{\underline{A}}{A}, \underline{\underline{B}}]_{+}=\underset{=}{A} \underline{\underline{B}}+\underline{\underline{B}} \underset{\underline{A}}{\mathrm{~A}}$ have been used to simplify the notation. The approximate character of this diabatization is revealed

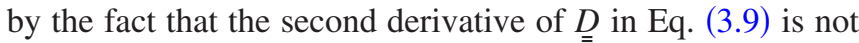
symmetric with respect to the components $a$ and $b$. If the electronic basis were complete, the asymmetry in the deriva- tives would be exactly zero. To remove this asymmetry for the quasidiabatization, we set

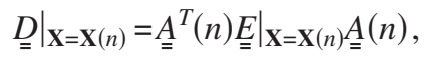

$$
\begin{aligned}
& \left.\frac{\partial}{\partial X_{a}} \stackrel{=}{ }\right|_{\mathbf{X}=\mathbf{X}(n)}=\left.\underline{A}^{T}(n)\left(\frac{\partial}{\partial X_{a}} \underline{\underline{E}}+[\underline{\underline{F}} a, \underline{\underline{E}}]_{-}\right)\right|_{\mathbf{X}=\mathbf{X}(n)} \underset{=}{A}(n), \\
& \frac{\partial^{2}}{\partial X_{b} \partial X_{a}}=\left.\right|_{\mathbf{X}=\mathbf{X}(n)}=\underline{A}^{T}(n)\left(\frac { 1 } { 2 } \left\{\left[\frac{\partial}{\partial X_{b}} \underline{\underline{F}}, \underline{\underline{E}}\right]_{-}\right.\right. \\
& +\left[\frac{\partial}{\partial X_{a}} \underline{F}_{b}, \underline{\underline{E}}\right]_{-}+\left[\underline{F}_{b} \underline{\underline{F}}_{a}, \underline{\underline{E}}\right]_{+} \\
& \left.+\left[\underline{F}_{a} \underline{\underline{F}}_{b}, \underline{\underline{E}}\right]_{+}\right\}+\left[\underline{\underline{F}}_{a}, \frac{\partial}{\partial X_{b}} \underline{\underline{E}}\right] \\
& +\left[\underline{\underline{F}}_{b}, \frac{\partial}{\partial X_{a}} \underline{\underline{E}}\right]_{-}-\underline{\underline{F}}_{a} \underline{\underline{E}} \underline{\underline{F}}_{b}-\underline{\underline{F}}_{b} \underline{\underline{E}} \underline{\underline{F}} a \\
& +\frac{\partial^{2}}{\partial X_{b} \partial X_{a}}=\left.\right|_{\mathbf{X}=\mathbf{X}(n)} \underset{=}{A}(n) \text {. }
\end{aligned}
$$

Now, it is important to note that the constant matrices $A_{=}^{T}(n)$ and $\underset{=}{A}(n)$ pre- and postmultiply all the terms on the righthand side of Eq. (3.10). Hence, we can define a new quantity $\tilde{D}$, such that

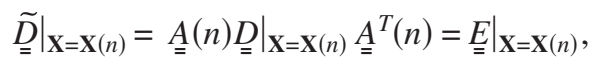

$$
\begin{aligned}
& \left.\frac{\partial}{\partial X_{a}} \tilde{=}\right|_{\mathbf{X}=\mathbf{X}(n)}=\underset{=}{A}(n) \frac{\partial}{\partial X_{a}}=\left.\right|_{\mathbf{X}=\mathbf{X}(n)} \stackrel{A}{A}^{T}(n), \\
& \left.\frac{\partial^{2}}{\partial X_{b} \partial X_{a}} \tilde{=}\right|_{\mathbf{X}=\mathbf{X}(n)}=\left.\underset{=}{A}(n) \frac{\partial}{\partial X_{b} \partial X_{a}} \underset{=}{D}\right|_{\mathbf{X}=\mathbf{X}(n)} \stackrel{A}{A}^{T}(n) .
\end{aligned}
$$

The value of $\widetilde{D}$ and the derivatives of $\tilde{D}$ with respect to the Cartesian coordinates are all known from $a b$ initio calculations. These derivatives can be transformed into derivatives with respect to the local internal coordinates of Eq. (3.6) by inverting (uniquely) the following equations:

$$
\begin{aligned}
\left.\frac{\partial}{\partial X_{a}} \tilde{D}\right|_{\mathbf{X}=\mathbf{X}(n)}= & \left.\sum_{k=1}^{3 N-6} \frac{\partial}{\partial \xi_{k}} \tilde{D}\right|_{\mathbf{X}=\mathbf{X}(n)} \mathbf{U}_{k a}(n) / \lambda_{k}, \\
\left.\frac{\partial^{2}}{\partial X_{b} \partial X_{a}} \tilde{D}\right|_{\mathbf{X}=\mathbf{X}(n)} & \left.\sum_{j=1}^{3 N-6} \sum_{k=1}^{3 N-6} \frac{\partial^{2}}{\partial \xi_{j} \partial \xi_{k}} \tilde{D}\right|_{\mathbf{X}=\mathbf{X}(n)} \\
& \times \frac{\mathbf{U}_{k a}(n) \mathbf{U}_{j b}(n)}{\lambda_{k} \lambda_{j}} \\
& +\left.\sum_{k=1}^{3 N-6} \frac{\partial}{\partial \xi_{k}} \tilde{D}\right|_{\mathbf{X}=\mathbf{X}(n)} \frac{\partial^{2} \xi_{k}}{\partial X_{b} \partial X_{a}} \mathbf{X = \mathbf { X } ( n )} .
\end{aligned}
$$

We solve Eq. (3.12) to obtain the local internal coordinate first and second derivatives of $\tilde{D}$. These derivatives provide the data needed to write a Taylor expansion for $\widetilde{D}$ which is well defined and is independent of translation and rotation of 
the molecule. From Eq. (3.11), a Taylor expansion for $\underset{\underline{D} \text { can }}{\text { can }}$ then be written as

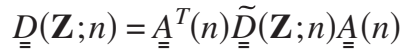

$$
\begin{aligned}
& =A_{\underline{A}}^{T}(n)\left[\tilde{\underline{D}}(n)+\left.\sum_{k=1}^{3 N-6} \frac{\partial}{\partial \xi_{k}} \tilde{\widetilde{D}}\right|_{(n)} \delta \xi_{k}\right. \\
& \left.+\left.\frac{1}{2} \sum_{j=1}^{3 N-6} \sum_{k=1}^{3 N-6} \frac{\partial^{2}}{\partial \xi_{j} \partial \xi_{k}} \tilde{D}\right|_{(n)} \delta \xi_{k} \delta \xi_{j}\right] \underset{=}{A}(n),
\end{aligned}
$$

where $\delta \xi_{k}=\xi_{k}-\xi_{k}(n)$. The importance of Eq. (3.13) is that the Taylor series $\widetilde{\mathbf{D}}(\mathbf{Z} ; n)$ can be evaluated from $a b$ initio data without any knowledge of the actual value of the ADT matrix at the data point. If $\underset{\equiv}{A}(n)$ is determined, then the local Taylor series for $\underset{D}{D(\mathbf{Z})}$ is determined. Our major remaining task is to determine $\underset{=}{A}(n)$. This task is aided by a knowledge of the symmetry of $\underset{\underline{A}}{A}(n), \widetilde{D}$, and $\underset{D}{D}$ under the operations of the CNPI group; so we first consider this symmetry.

\section{Symmetry}

The DPEM can change in nontrivial ways on permutation of indistinguishable atoms or on inversion. This is because the ADT matrix can change on permutation,

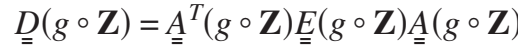

$$
\begin{aligned}
& =\underline{\underline{A}}^{T}(g \circ \mathbf{Z}) \underline{\underline{E}}(\mathbf{Z}) \underset{\underline{A}}{A}(g \circ \mathbf{Z}) .
\end{aligned}
$$

To understand how $\underset{\underline{A}}{A}(\mathbf{Z})$ and $\underset{\underline{D}}{D(\mathbf{Z})}$ transform under the CNPI group, we begin by considering the transformation of the related adiabatic quantities.

\section{Permutation of adiabatic quantities}

Away from a conical intersection, the adiabatic wave functions are uniquely determined to within a sign,

$$
g \circ \underline{\psi}(\mathbf{x}, \mathbf{X})=\underline{\underline{S}}(g) \underline{\psi}(\mathbf{x}, \mathbf{X}),
$$

where $\underset{\underline{S}}{S}(g)$ is a diagonal matrix of \pm 1 's. This determines how the derivative couplings transform under the CNPI group,

$$
g \circ \underline{\underline{F}}_{i}(\mathbf{X})=\underline{\underline{S}}(g) \underline{\underline{F}}_{g \circ i}(\mathbf{X}) \underset{\underline{S}}{(g)},
$$

where the $g \circ i$ subscript denotes that the Cartesian components of the derivative coupling vectors have been permuted. In general, the $\underset{\underline{S}}{S}(\mathrm{~g})$ matrices form a double-valued group and depend on the location of the electronic degeneracies.

\section{Permutation of diabatic quantities}

We begin by considering how the adiabatic electronic wave functions at a conical intersection transform under symmetry operations. This allows us to understand how the corresponding diabatic wave functions transform.

If there is an electronic ndegen-fold degeneracy between a subset of the electronic states at $\mathbf{X}=\mathbf{Y}$ (ndegen $\leqslant$ nstates), then this subset of electronic wave functions is not uniquely defined. Any unitary transformation of these ndegen orthogonal wave functions is also an eigenbasis of ndegen-fold degenerate wave functions. The CNPI operations do not lift the degeneracy, since the electronic Hamiltonian is invariant under the CNPI operations. This means that the ndegen wave functions form a representation of the CNPI group. If $\mathbf{Y}$ corresponds to a point-group symmetric geometry $(\exists g \neq I$ such that $g \circ \mathbf{Y}=\mathbf{Y}$ ), the electronic wave functions also transform as a representation of the point group:

$$
g \circ \psi_{n}(\mathbf{x}, \mathbf{Y})=\sum_{k=1}^{\text {ndegen }} \hat{M}_{k, n}(g) \psi_{k}(\mathbf{x}, \mathbf{Y}),
$$

where $\hat{M}_{k, n}(g)$ is an ndegen $\times$ ndegen unitary matrix. In a matrix notation,

$$
g \circ \underline{\psi}(\mathbf{x}, \mathbf{Y})=\hat{\underline{M}}(g)^{T} \underline{\psi}(\mathbf{x}, \mathbf{Y}) .
$$

If nstates $\rightarrow \infty$, the diabatic wave functions at an arbitrary geometry $\mathbf{X}$ are related to the adiabatic wave functions at the conical intersection $\mathbf{Y}$ :

$$
\widetilde{\psi}^{\infty}(\mathbf{x}, \mathbf{X})=\left[\underline{\underline{A}}^{\infty}(\mathbf{Y})\right]^{T} \underline{\psi}^{\infty}(\mathbf{x}, \mathbf{Y})
$$

Then,

$$
\begin{aligned}
g \circ \tilde{\psi}^{\infty}(\mathbf{x}, \mathbf{X}) & =\left[\underline{A}^{\infty}(g \circ \mathbf{Y})\right]^{T} g \circ \underline{\psi}^{\infty}(\mathbf{x}, \mathbf{Y}) \\
& =\left[\underline{A}^{\infty}(\mathbf{Y})\right]^{T}\left[\hat{\underline{M}}^{\infty}(g)\right]^{T} \underline{\psi}^{\infty}(\mathbf{x}, \mathbf{Y}) \\
& =\left[\underline{A}^{\infty}(\mathbf{Y})\right]^{T}\left[\hat{\underline{M}}^{\infty}(g)\right]^{T} \underline{A}^{\infty}(\mathbf{Y})\left[\underline{A}^{\infty}(\mathbf{Y})\right]^{T} \underline{\psi}^{\infty}(\mathbf{x}, \mathbf{Y}) \\
& =\left[\underline{\underline{A}}^{\infty}(\mathbf{Y})\right]^{T}\left[\hat{\underline{M}}^{\infty}(g)\right]^{T} \underline{\underline{A}}^{\infty}(\mathbf{Y}) \tilde{\psi}^{\infty}(\mathbf{x}, \mathbf{X}) \\
& \equiv\left[\underline{\underline{M}}^{\infty}(g)\right]^{T} \underline{\psi}^{\infty}(\mathbf{x}, \mathbf{X}) .
\end{aligned}
$$

If we let $M(g)$ represent the relevant nstates $\times$ nstates block of $M_{=}^{\infty}(g)$, then for the nstates diabatic wave functions

$$
g \circ \tilde{\psi}(\mathbf{x}, \mathbf{X})=[\underline{\underline{M}}(g)]^{T} \tilde{\psi}(\mathbf{x}, \mathbf{X}) .
$$

If we determine the symmetry of the adiabatic wave functions at the conical intersection, we can determine the symmetry of the diabatic wave functions globally.

The symmetry of the ADT matrix at an arbitrary geometry can now be derived. Using Eqs. (3.15) and (3.21), we have

$$
\begin{aligned}
& g \circ \tilde{\psi}(\mathbf{x}, \mathbf{X})=g \circ \underline{\underline{A}}^{T}(\mathbf{X}) g \circ \underline{\psi}(\mathbf{x}, \mathbf{X}) \\
& \therefore[\underline{\underline{M}}(g)]^{T} \underline{\underline{A}}^{T}(\mathbf{X}) \underline{\psi}(\mathbf{x}, \mathbf{X})=g \circ \underline{A}^{T}(\mathbf{X}) \underline{\underline{S}}(g) \underline{\psi}(\mathbf{x}, \mathbf{X}) .
\end{aligned}
$$

As $\underline{\underline{S}}^{T}(g)=\underline{\underline{S}}(g)$ we have

$$
g \circ \underset{=}{A}(\mathbf{X})=\underset{=}{S}(g) \underset{=}{A}(\mathbf{X}) \underset{=}{M}(g) .
$$

This tells us how the ADT matrix is transformed at a general geometry $\mathbf{X}$. From this we can determine how the elements of the diabatic potential matrix are transformed under the CNPI group

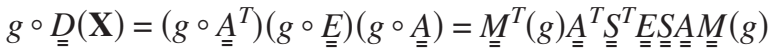

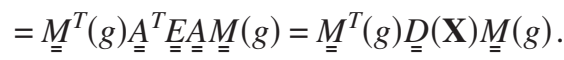

This shows that the DPEM is not an invariant of the CNPI group, in contrast to a simple PES, but transforms in a fash- 
ion determined by the degenerate adiabatic electronic wave functions. An automated numerical method for deriving the symmetry matrices $\underline{\underline{M}}(g)$ will be discussed below. Since the DPEM is evaluated using local Taylor series approximations, the symmetry transformations of the terms in these Taylor series must also be understood.

\section{Transformation of the Taylor series}

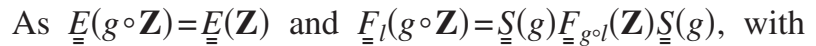
some algebra it can be shown that the Taylor series coefficients for $\underset{D}{\tilde{D}}$ are transformed as

$$
\begin{aligned}
& \left.\underline{\underline{D}}\right|_{g \circ \underline{\underline{X}}(n)}=\left.\underline{\underline{E}}\right|_{\underline{\underline{X}}(n)}, \\
& \left.\frac{\partial}{\partial X_{l}} \underline{\tilde{D}}\right|_{g \circ \underline{\underline{X}}(n)}=\left.\underset{\underline{S}}{ }(g) \frac{\partial}{\partial X_{g \circ l}} \tilde{\underline{D}}\right|_{\underline{\underline{X}}(n)} \underline{\underline{S}}(g), \\
& \left.\frac{\partial^{2}}{\partial X_{k} \partial X_{l}} \tilde{\underline{D}}\right|_{g \circ \underline{\underline{X}}(n)}=\left.\underline{\underline{S}}(g) \frac{\partial^{2}}{\partial X_{g \circ k} \partial X_{g \circ l}} \underline{\tilde{D}}\right|_{\underline{\underline{X}(n)}} \underline{S}(g) .
\end{aligned}
$$

Therefore, if we act on all the terms in $\underset{\underline{D}}{\widetilde{w}}$ with a CNPI transformation, we have

$$
\begin{aligned}
& \tilde{\underline{D}}(g \circ \mathbf{X} ; g \circ n)=\left.\tilde{D}\right|_{g \circ \mathbf{X}(n)}+\left.\sum_{l=1}^{\text {nbond }} \frac{\partial}{\partial X_{l}} \underline{\tilde{D}}\right|_{g \circ \mathbf{X}(n)}\left(g \circ\left[X_{l}-X_{l}(n)\right]\right)+\left.\sum_{l, k=1}^{\text {nbond }} \frac{\partial^{2}}{\partial X_{k} \partial X_{l}} \tilde{\underline{D}}\right|_{g \circ \mathbf{X}(n)}\left(g \circ\left[X_{l}-X_{l}(n)\right]\right)\left(g \circ\left[X_{k}-X_{k}(n)\right]\right)=\left.\underline{\underline{D}}\right|_{\mathbf{X}(n)}
\end{aligned}
$$

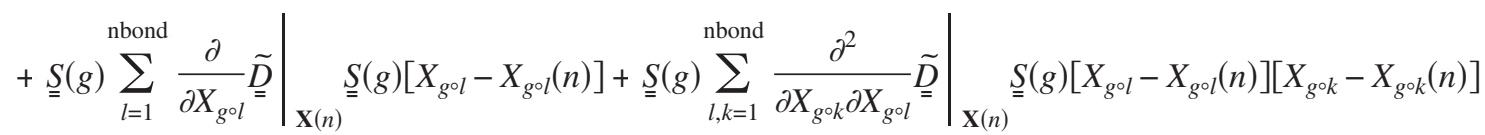

$$
\begin{aligned}
& =\underline{\underline{S}}(g) \underset{\underline{D}}{\tilde{D}}(\mathbf{X} ; n) \underline{\underline{S}}(g) \text {. }
\end{aligned}
$$

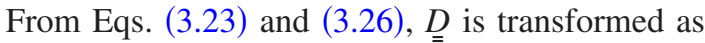

$$
\begin{aligned}
\underline{\underline{D}}(g \circ \mathbf{X} ; g \circ n)= & \underline{\underline{A}}^{T}(g \circ n) \underline{\underline{\underline{D}}}(g \circ \mathbf{X} ; g \circ n) \underline{\underline{A}(g \circ n)} \\
= & {\left[\underline{\underline{M}}^{T}(g) \underline{\underline{A}}^{T}(n) \underline{\underline{S}}(g)\right] \underline{\underline{S}}(g) \underline{\underline{D}}(\mathbf{X} ; n) \underline{\underline{S}}(g) } \\
& \times[\underline{\underline{S}}(g) \underline{\underline{A}}(n) \underline{\underline{M}}(g)] \\
= & \underline{\underline{M}}^{T}(g) \underline{\underline{A}}^{T}(n) \tilde{\underline{D}}(\mathbf{X} ; n) \underline{\underline{A}}(n) \underline{\underline{M}}(g) \\
= & \underline{\underline{M}}^{T}(g) \underline{\underline{D}}(\mathbf{X} ; n) \underline{\underline{M}}(g) .
\end{aligned}
$$

This implies that

$$
\begin{aligned}
\underline{\underline{D}}(\mathbf{X} ; g \circ n) & =g \circ \underline{\underline{D}}\left(g^{-1} \circ \mathbf{X} ; n\right) \\
& =\underline{\underline{M}}^{T}(g) \underline{\underline{D}}\left(g^{-1} \circ \mathbf{X} ; n\right) \underline{\underline{M}}(g) .
\end{aligned}
$$

Hence, to evaluate a Taylor series about a transformed data point, $g \circ n$, we can simply evaluate the Taylor series about the original data point, at $g^{-1} \circ \mathbf{X}$, and multiply by the appropriate symmetry matrices. This approach requires that we know the identity of the inverse transformations. via

However, we can also evaluate the Taylor series directly

$$
\begin{aligned}
& \underline{\underline{D}}(\mathbf{X} ; g \circ n)=\underline{\underline{A}}^{T}(g \circ n) \underset{\underline{D}}{\widetilde{X}}(\mathbf{X} ; g \circ n) \underline{\underline{A}}(g \circ n) \\
& =\left[\underline{\underline{M}}^{T}(g) \underline{\underline{\underline{A}}}^{T}(n) \underline{\underline{S}}(g)\right] \underline{\underline{D}}(\mathbf{X} ; g \circ n)[\underline{\underline{S}}(g) \underline{\underline{A}}(n) \underline{\underline{M}}(g)] \\
& =\underline{\underline{M}}^{T}(g) \underline{\underline{A}}^{T}(n)[\underline{\underline{S}}(g) \underline{\underline{D}}(\mathbf{X} ; g \circ n) \underline{\underline{S}}(g)] \underline{\underline{A}}(n) \underline{\underline{M}}(g) \text {. }
\end{aligned}
$$

From Eq. (3.25),

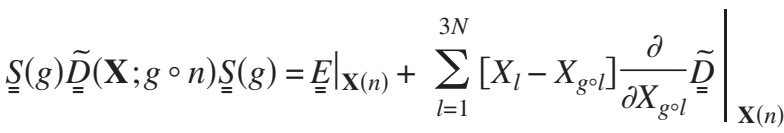

$$
\begin{aligned}
& +\frac{1}{2} \sum_{l=1}^{3 N} \sum_{k=1}^{3 N}\left[X_{l}-X_{g^{\circ} l}\right] \\
& \times\left.\left[X_{k}-X_{g \circ k}\right] \frac{\partial^{2}}{\partial X_{g \circ k} \partial X_{g \circ l}} \tilde{D}\right|_{\mathbf{X}(n)} .
\end{aligned}
$$

We must express this Taylor series in internal coordinates. We note that local internal coordinates near $g \circ \mathbf{X}(n)$ are defined by

$$
\xi_{k}^{(g \circ n)}=\sum_{\alpha=1}^{\substack{\text { nbond } \\+ \text { ndotc }}} U_{k, g \circ \alpha}(n) Z_{\alpha} / \lambda_{k}, \quad k=1, \ldots, 3 N-6 .
$$

Note also that

$$
\begin{gathered}
\xi_{k}^{(g \circ n)}[\mathbf{Z}=g \circ \mathbf{Z}(n)]=\xi_{k}[\mathbf{Z}(n)]=\xi_{k}(n), \\
\left.\frac{\partial}{\partial \xi_{k}} \tilde{D}\right|_{(g \circ n)}=\left.\frac{\partial}{\partial \xi_{k}} \tilde{D}\right|_{(n)}, \\
\left.\frac{\partial^{2}}{\partial \xi_{j} \partial \xi_{k}} \tilde{D}\right|_{(g \circ n)}=\left.\frac{\partial^{2}}{\partial \xi_{j} \partial \xi_{k}} \tilde{D}\right|_{(n)} .
\end{gathered}
$$

Hence, from Eqs. (3.29)-(3.32), we have 


$$
\begin{aligned}
\underline{\underline{D}(\mathbf{Z} ; g \circ n)=} & \underline{\underline{M}}^{T}(g) \underline{\underline{A}}^{T}(n)\left\{\underline{\underline{E}}(n)+\left.\sum_{k=1}^{3 N-6} \frac{\partial}{\partial \xi_{k}} \tilde{\underline{D}}\right|_{(n)}\right. \\
& \times\left[\xi_{k}^{(g \circ n)}(\mathbf{Z})-\xi_{k}(n)\right] \\
& +\left.\frac{1}{2} \sum_{k=1}^{3 N-6} \sum_{j=1}^{3 N-6} \frac{\partial^{2}}{\partial \xi_{k} \partial \xi_{j}} \underline{\tilde{D}}\right|_{(n)}\left[\xi_{k}^{(g \circ n)}(\mathbf{Z})-\xi_{k}(n)\right] \\
& \left.\times\left[\xi_{j}^{(g \circ n)}(\mathbf{Z})-\xi_{j}(n)\right]\right\} \underline{\underline{\underline{A}}(n) \underline{\underline{M}}(g)}
\end{aligned}
$$

\section{Assigning the ADT matrices}

The formula for all the individual Taylor series for all symmetry related data points has been derived. The unknown quantity in this formula is the ADT matrix at each unique data point $\underset{\underline{A}}{A}(n)$. The method used to evaluate the $\underset{\underline{A}}{\underline{a} \text { matrices }}$ for all data points is long and complicated. As well as assigning values to each $A(n)$, the signs of the derivative couplings at each data point must be determined, since the signs are undetermined from the $a b$ initio calculations. For ease of understanding, all the important details are presented in Appendix A, with necessary derivations in the following appendixes, and a qualitative description is presented here.

The basic strategy used to evaluate the $A$ matrices is to demand that the whole set of ADT matrices, for all data points and their CNPI transformations, is mutually consistent. If a data point $\mathbf{Z}(n)$ is close to another data point, say, $g \circ \mathbf{Z}(m)$, then both points are close to their midpoint, $\mathbf{Z}_{\text {mid }}$.

$$
\mathbf{Z}_{\text {mid }}=\frac{1}{2}[\mathbf{Z}(n)+g \circ \mathbf{Z}(m)] \text {. }
$$

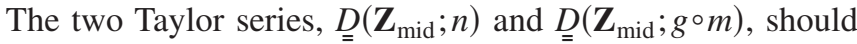
be nearly equal:

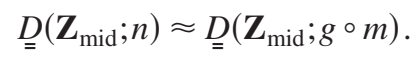

If $\underset{\underline{A}}{A}(\mathrm{~g} \circ \mathrm{m})$ is known, then $\underset{\underline{A}}{(n)}(n)$ and the signs of the derivative couplings can be estimated by minimizing $\delta D$,

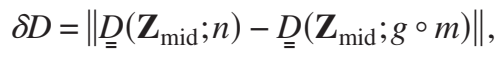

with respect to variations in $A(n)$ and the signs of the derivative couplings. Even better, if the $A$ matrices are known at many data points that are close to $\mathbf{Z}(n)$, then a more accurate estimate of $\underset{\underline{A}}{A}(n)$ and the relevant signs can be obtained from a weighted average of the estimates based on each neighboring data point, closer data points having higher weights.

To begin this process, we are free to fix the $\underline{\underline{A}}$ matrix at some arbitrary geometry and to fix the signs of the derivative couplings there. We take the $\underset{A}{A}$ matrix to be the identity near a configuration, $\mathbf{Z}(1)$, which is nearly symmetric under at least one CNPI operation and which is close to a conical intersection (see Appendix A). We can then use Eq. (3.35), with $n=m=1$, to evaluate the symmetry matrices numerically (see Appendix F).

The $A$ matrix for the data point [call it $\mathbf{Z}(2)$ ], closest to $g \circ \mathbf{Z}(1)$ for some $g$, is then determined by mutual consistency as above. Both data points are then used with appropriate weights to determine $A(3)$. This propagative procedure is continued until all the $\underset{\underline{A}}{A}$ matrices have been estimated.
Finally, we take these $A$ matrices as the initial values in a simultaneous minimization of the suitably weighted errors, as in Eq. (3.36), at the midpoints of all neighboring data points. This produces a set of mutually consistent $A$ matrices and assigned signs for the derivative couplings. Loosely speaking, we have constructed an approximate global integration of the derivative couplings by demanding that the

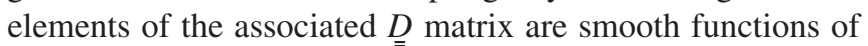
the nuclear coordinates.

\section{E. The weights}

The weights $w(\mathbf{Z} ; g \circ n)$ in the interpolation formula (3.1), and elsewhere, are used to approximate a function value at $\mathbf{Z}$ by a weighted average of local approximations. These local approximations are based on CNPI variants $(g \in G)$ of data points, $n=1, \ldots$,ndata. The weights have the following properties:

$$
\sum_{g \in G} \sum_{n=1}^{\text {ndata }} w(\mathbf{Z} ; g \circ n)=1
$$

and

$$
w(\mathbf{Z}(n) ; i)=\delta_{n, i} .
$$

From (3.37), we see that Eq. (3.1) is a weighted average of local approximations. Eq. (3.38) ensures that Eq. (3.1) is an interpolation. The weights are constructed by normalizing the primitive weights, $\nu(\mathbf{Z}, n)$,

$$
w(\mathbf{Z} ; n)=\frac{\nu(\mathbf{Z} ; n)}{\sum_{g \in G} \sum_{m=1}^{\text {ndata }} \nu(\mathbf{Z} ; g \circ m)} .
$$

The primitive weights are designed to satisfy two conditions:

$$
\lim _{\mathbf{Z} \rightarrow \mathbf{Z}(n)} \nu(\mathbf{Z} ; n) \rightarrow \infty,
$$

and

$$
\lim _{\|\mathbf{Z}-\mathbf{Z}(n)\| \rightarrow \infty} \nu(\mathbf{Z} ; n)=0 .
$$

Equation (3.41) enforces locality; that is, only data points that are close to $\mathbf{Z}$ contribute significantly to the weighted sum.

A "one-part" primitive weight function which satisfies these conditions is given by ${ }^{16,22}$

$$
\nu(\mathbf{Z} ; n)=\frac{1}{\left(\sum_{l=1}^{\text {nbond+ndotc }}\left(Z_{l}-Z_{l}(n)\right)^{2}\right)^{p}},
$$

where $2 p>3 N-3$ ensures that the contribution from distant data points is negligible. While the singularity in $\nu(\mathbf{Z}, n)$ can be removed from the numerator and denominator of $\mathrm{Eq}$. (3.39), in practice we find that simple evaluation of Eq. (3.39) is numerically well behaved whether $\mathbf{Z}$ is close to or far from $\mathbf{Z}(n)$, when using double precision arithmetic. 
A more accurate primitive weight function, called a "two-part" weight function, is given by

$$
\begin{aligned}
\nu(Z ; n)= & \left\{\left[\sum_{l=1}^{\text {nbond+ndotc }}\left(\frac{Z_{l}-Z_{l}(n)}{d_{l}(n)}\right)^{2}\right]^{q}\right. \\
& \left.+\left[\sum_{l=1}^{\text {nbond+ndotc }}\left(\frac{Z_{l}-Z_{l}(n)}{d_{l}(n)}\right)^{2}\right]^{p}\right\}^{-1},
\end{aligned}
$$

where $q \geqslant 2$. The $d_{l}(n)$ denote confidence lengths about the $n$th data point for each direction $l$ in space. When $\mathbf{Z}$ is within the "confidence volume" of $\mathbf{Z}(n)$, the power $q$ dominates and the weights change slowly with $\mathbf{Z}$; outside the confidence volume, the power $p$ dominates and the primitive weight decays rapidly as $\|\mathbf{Z}-\mathbf{Z}(n)\|$ increases.

The confidence lengths are determined by a Bayesian analysis of the measured error in the gradient of the DPEM for a given Taylor series. ${ }^{19}$ This error can be measured at neighboring data points, even when the ADT matrices are not known. The details are presented in Appendix E, and the confidence lengths $d_{l}(n)$, are given by Eq. (E9).

\section{F. Automated evaluation of the symmetry matrices}

In Sec. III C, the symmetry matrices were determined by considering the way the adiabatic electronic wave functions at a conical intersection transform under the CNPI group operations. This consideration involves inspection of the electronic wave functions in some detail. However, the construction of the DPEM should be as automated as possible. Hence, it is useful to develop a method whereby the symmetry matrices are evaluated numerically in an automated process which does not require "manual" intervention. Appendix $\mathrm{F}$ presents an algorithm for automatically constructing the group symmetry matrices. The method uses only ab initio data and Taylor series expansions of the DPEM.

The symmetry matrices are evaluated numerically. The numerical accuracy of the matrices is improved if $a b$ initio data are available near a conical intersection where the derivative coupling is large in magnitude. However, these matrices cannot be exact. At present, the "user" must intervene at the end of the process to convert the matrices to numerically exact values. For example, in the $\mathrm{NH}_{3}$ system considered below, an element of a symmetry matrix may have a value of $0.86743 \ldots$ This can be recognized as an approximation to $\sqrt{3} / 2$. At the present stage of the development of this approach, maintaining the exact symmetry for the DPEM requires such manual corrections to the numerical symmetry matrices or algebraic evaluation of the matrices from consideration of the adiabatic electronic wave functions. Of course, the symmetry matrices need only be determined once, at the beginning of the DPEM construction process.

\section{G. Summary}

To summarize the entire interpolation procedure, (1) the DPEM is evaluated from Eq. (3.1), (2) the weight function in (3.1) is given by Eqs. (3.39), (3.43), and (E9), and (3) the Taylor series in Eq. (3.1) are given by Eq. (3.33). In that equation, the internal coordinates are defined by Eqs. (3.4)-(3.6) which have the symmetry defined by Eq. (3.32). The $\underline{\underline{M}}(g)$ matrices are derived in Appendix F. The $\underline{\underline{A}(n)}$ matrices are assigned by the procedure of Sec. III D and Appendix A.

\section{CONSTRUCTION PROCEDURE}

\section{A. The iterative scheme}

One important aspect of the interpolation formula [Eq. (3.1)] has yet to be discussed, namely, the positions of the data points $\mathbf{Z}(n)$. These positions are determined by an iterative scheme which is essentially that used to construct an adiabatic PES.

We assume that some initial investigation of the ab initio quantum chemistry of the system has been carried out. This is essential to determine the level of $a b$ initio theory which is necessary to provide chemically reliable estimates of the molecular energies in relevant regions of the configuration space. It may be that some critical saddle points and equilibrium structures have been found, along with approximate pathways connecting them. In the case of nonadiabatic processes, it is reasonable to assume that at least the approximate location of a relevant conical intersection has been determined; or else we might not be considering the need for a diabatic potential matrix rather than a simple PES. The following is the construction procedure.

(1) Include in an initial data set the approximate conical intersection configuration, any known stationary points on the adiabatic PESs, and perhaps tens of configurations on the paths which connect these points.

(2) Perform the necessary ab initio calculations for the nstate adiabatic energies, first and second derivatives of the energies, and interstate derivative couplings. Stateaveraged multiconfiguration self-consistent-field (MC$\mathrm{SCF}$ ) or multireference configuration interaction (MRCI) calculations are required. Convert the Cartesian derivatives into derivatives with respect to local internal coordinates.

(3) Beginning with a configuration where the derivative couplings are large assign the ADT matrices for the whole data set. Determine the symmetry matrices and confidence lengths.

(4) Simulate the dynamics of interest with a small number of classical trajectories evaluated using the current data set. Use surface hopping trajectories for nonadiabatic processes, as detailed below.

(5) Select one of the molecular configurations encountered in these trajectories as a new data point, using the criteria discussed below.

(6) Repeat from (2) above.

As this iteration is repeated, the size of the data set increases. Periodically, the iteration is interrupted and an accurate simulation of the dynamics is carried out. This may be via a large scale classical simulation or by solution of the 
Schrödinger equation in the diabatic representation. When the results of these simulations do not change significantly with change in the data set size, the DPEM is taken to be converged.

\section{B. Surface hopping}

During the DPEM construction process, we employ classical surface hopping dynamics to sample the dynamically relevant region of configuration space. This avoids the very substantial computational cost of repeated quantum simulations of the diabatic dynamics. Ultimately, it must be demonstrated that the DPEM constructed with this classical sampling are converged for quantum simulations.

The trajectory surface hopping (TSH) method developed by Tully, which is based on the fewest switches algorithm, is used in the calculations presented here. ${ }^{23}$ It would be useful to investigate the effect of using other surface hopping or semiclassical methods ${ }^{24}$ on the distribution of data points, but the simple Tully method has been employed in the first instance. A brief description of the nuclear equations of motion is presented here. The nuclear coordinates obey the classical equations for motion on a single PES:

$$
\begin{aligned}
& m_{i} \dot{X}_{i}=P_{i}, \\
& \dot{P}_{i}=\frac{\partial E_{k}}{\partial X_{i}} .
\end{aligned}
$$

$E_{k}$ refers to the PES of the $k$ th adiabatic electronic state, $X_{i}$ are the atomic Cartesian coordinates, and $P_{i}$ are the atomic Cartesian momenta. The identity of the electronic state "occupied" at any instant in time is identified by evaluating the time evolution of this "occupancy" as follows.

The time-dependent Schrödinger equation for the electronic wave function is given by

$$
i \hbar \frac{\partial}{\partial t} \Psi(\mathbf{x}, \mathbf{X} ; t)=H \Psi(\mathbf{x}, \mathbf{X} ; t) .
$$

Expanding $\Psi(\mathbf{x}, \mathbf{X} ; t)$ in the adiabatic representation, where $C_{j}(t)$ are complex-valued expansion coefficients and $\psi_{j}$ are the adiabatic wave functions, we get

$$
\Psi=\sum_{j=1}^{\text {nstates }} C_{j}(t) \psi_{j}[\mathbf{x}, \mathbf{X}(t)] .
$$

Inserting Eq. (4.3) into Eq. (4.2), multiplying from the left by $\psi_{i}^{*}$, and integrating over the electronic coordinates gives

$$
i \hbar \frac{d C_{k}}{d t}=E_{k} C_{k}-i \hbar \sum_{n=1}^{\text {nstates }} C_{n} \mathbf{F}_{k, n} \cdot \dot{\mathbf{X}}
$$

where $\mathbf{F}_{k, n}=\left\langle\psi_{k} \mid \nabla \psi_{n}\right\rangle$ is the derivative coupling matrix element.

We define the density matrix element $a_{k, j}=C_{k} C_{j}^{*}$. From Eq. (4.4), we have

$i \hbar \dot{a}_{k, j}=\left(E_{k}-E_{j}\right) a_{k, j}-i \hbar \sum_{n=1}^{\text {nstates }} a_{j, n}^{*} \mathbf{F}_{k, n} \cdot \dot{\mathbf{X}}+a_{k, n} \mathbf{F}_{j, n} \cdot \dot{\mathbf{X}}$.
The set of coupled differential equations, (4.1) and (4.5), are numerically integrated throughout the trajectory. The population of the $k$ th state is given by $a_{k, k}$. After each time step the probabilities of hopping from the current state $k$ to a state $j$ is computed by considering the quantity

$$
g_{k, j}=\frac{-2\left(a_{k, j}+a_{k, j}^{*}\right) \mathbf{F}_{k, j} \cdot \dot{\mathbf{X}}}{\left(a_{k, k}+a_{k, k}^{*}\right)} \Delta t .
$$

A uniform random number $\mu$ between 0 and 1 is then generated, and a switch between states $k$ and $j$ will be invoked if

$$
g_{k, j}>\mu
$$

and if there is enough kinetic energy to allow the switch. When a hop occurs, the nuclear potential energy $E_{k}$ is replace by $E_{j}$, and the nuclear kinetic energy is adjusted to ensure the total (potential+kinetic) is unchanged (a hop from state $k$ to state $j$ is not affected if the total energy of the nuclei cannot be conserved). When a switch between states occurs, the adjustment of the nucleus momenta on the new PES is done using the nonadiabatic derivative coupling vector. ${ }^{25}$

\section{Derivative coupling}

The derivative couplings at each configuration in a trajectory are evaluated from the interpolated DPEM as fol-

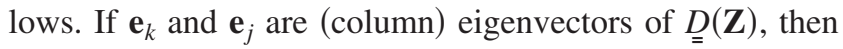

$$
\left(\mathbf{F}_{k, j}\right)_{\alpha}=\frac{\mathbf{e}_{k}^{T}\left(\partial \underline{\underline{D}} / \partial X_{\alpha}\right) \mathbf{e}_{j}}{E_{k}-E_{j}} .
$$

The arbitrary signs of the eigenvectors $\mathbf{e}_{k}$ and $\mathbf{e}_{j}$ are forced to be consistent from one time step to the next in the classical trajectories.

\section{TEST RESULTS}

In order to test the accuracy of an interpolated DPEM, we have used this approach to reproduce an analytic functional form, ${ }^{7}$ for the diabatic potential matrix for the three lowest electronic states of $\mathrm{NH}_{3}^{+}$. The advantages of this "test model" are that the energies and couplings at each data point are easily calculated so that extensive testing is facile, the interpolated diabatic energies can be compared with the analytic values, and the results of dynamics performed on the interpolated and the analytic potentials are easily compared. Hence, the convergence of the interpolation process is easily monitored. It must be noted, however, that this test does not examine the effect of nonremovable couplings. An examination of the robustness of this approach to the effects of nonremovable couplings is the subject of further work.

\section{A. Computational details}

The analytic surface ${ }^{7}$ for $\mathrm{NH}_{3}^{+}$was developed to study the dynamics in three electronic states following photoionization:

$$
\mathrm{NH}_{3}+h \nu \rightarrow \mathrm{NH}_{3}^{+}+e^{-} .
$$

State 3 (the second excited state of $\mathrm{NH}_{3}^{+}$) has a conical intersection with state 2 (the first excited state) along the $C_{3}$ symmetry axis. State 2 has a conical intersection with state 1 (the 
ground state) at a large angle of the umbrella mode.

For the simulation of $\mathrm{NH}_{3}^{+}$considered here, we assume that the distribution of initial $(t=0)$ positions and velocities of the atoms corresponds to the ground state of the neutral $\mathrm{NH}_{3}$, but that electronic state 3 of the cation is populated. The initial positions and velocities are sampled from a microcanonically distributed vibrational energy of 33 mhartree (approximately the zero point energy of $\mathrm{NH}_{3}$ ) above the ground state equilibrium geometry. The neutral PES was given by a modified Shepard interpolation with one unique (equilibrium) data point at the MP2/6-311G $(d, p)$ level of $a b$ initio theory, constructed with the GROW program. ${ }^{2}$ The surface hopping trajectories were integrated using the adaptive stepsize control Richardson extrapolation and the BulirschStoer method (see Ref. 26, p. 563). The population of the different states was monitored along the trajectory for $150 \mathrm{fs}$.

To construct the interpolated DPEM, an initial set of ten data points were spread between the bent geometry (equilibrium of the neutral $\mathrm{NH}_{3}$ ) and the planar equilibrium geometry of $\mathrm{NH}_{3}^{+}$. The energies and derivative couplings at each geometry were evaluated from the analytic surfaces and were used to construct the data set for the interpolation, as described above. At each subsequent iteration in the construction process, a new data point was selected from molecular configurations encountered in ten surface hopping trajectories. The data points were chosen by alternating the $h$-weight-based method and two variations on the variance sampling method, ${ }^{2}$ as follows. At regular intervals of time steps in each trajectory, we evaluate the variance of the DPEM, $\sigma^{2}$,

$$
\begin{aligned}
\sigma^{2}(\mathbf{Z})= & \sum_{n=1}^{\text {nstates }} \sum_{m=1}^{\text {nstates }}\left\{\sum_{g \in G} \sum_{n=1}^{\text {ndata }} w(\mathbf{Z} ; g \circ n)\right. \\
& \left.\times\left[D_{n, m}(\mathbf{Z} ; g \circ n)-D_{n, m}(\mathbf{Z})\right]^{2}\right\},
\end{aligned}
$$

and the variance in the gradient of the DPEM, $d \sigma^{2}$,

$$
\begin{aligned}
d \sigma^{2}(\mathbf{Z})= & \sum_{n=1}^{\text {nstates }} \sum_{m=1}^{\text {nstates nbond+ndoic }} \sum_{\alpha=1}\left\{\sum_{g \in G} \sum_{n=1}^{\text {ndata }} w(\mathbf{Z} ; g \circ n)\right. \\
& \left.\times\left[\frac{\partial\left[D_{n, m}(\mathbf{Z} ; g \circ n)-D_{n, m}(\mathbf{Z})\right]}{\partial Z_{\alpha}}\right]^{2}\right\} .
\end{aligned}
$$

Large values of $\sigma^{2}$ and $d \sigma^{2}$ indicate configurations where the interpolation for the DPEM and its gradient are uncertain. The variance sampling method used here involves choosing a new data point as the trajectory configuration with the largest value of $\sigma^{2}$ or (at the next iteration) the largest value of $d \sigma^{2}$.

\section{B. Results}

For data sets of 10, 50, 200, and 300 points, 1500 trajectories were run on the interpolated surfaces, and the average state populations as functions of time were calculated. To compare with these results, 40000 trajectories were run under the same conditions using the analytic surfaces. From
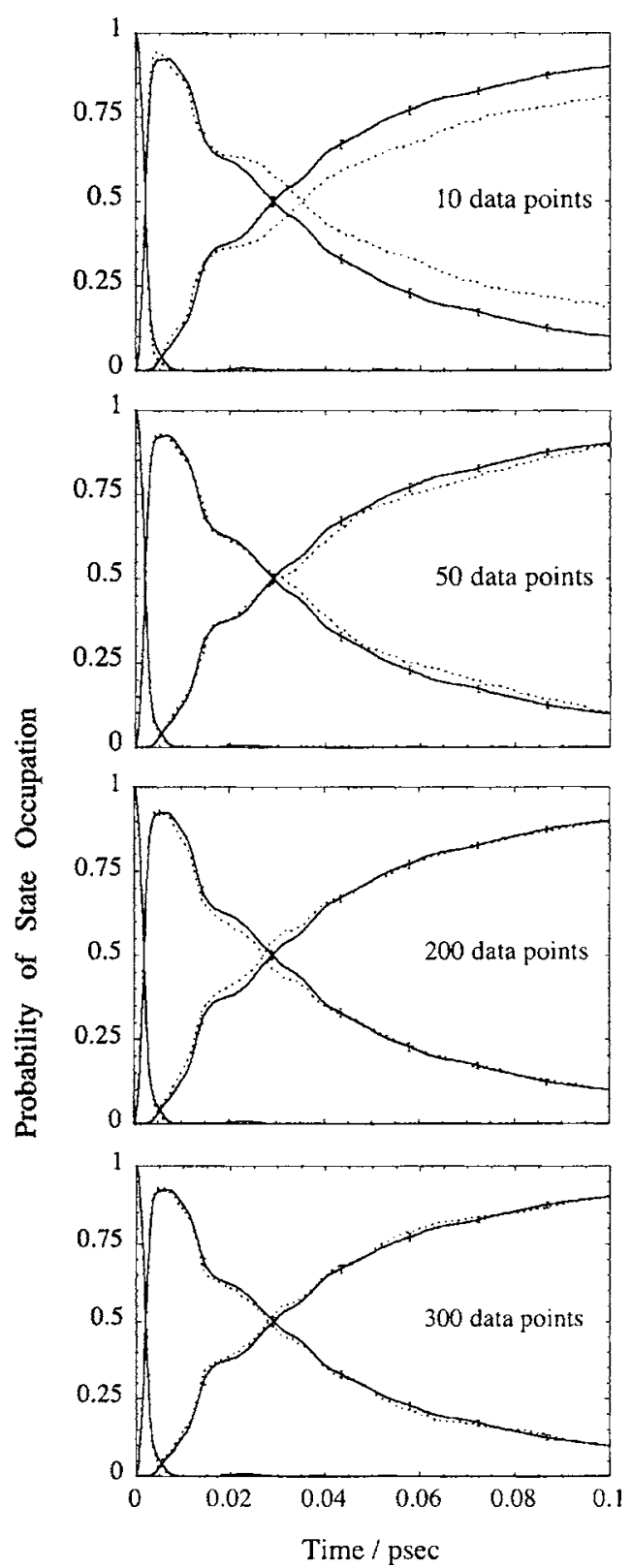

FIG. 1. The probabilities of occupation of electronic states 1, 2, and 3 of $\mathrm{NH}_{3}^{+}$are shown as a function of time. The solid lines denote the results of surface hopping dynamics on the analytic surface of Ref. 6 . The dashed lines denote the corresponding results from interpolation of the DPEM with the number of data points shown. The probability for state 3 decays from 1 at short times, while the probability for state 2 rises initially before falling. The error bars on the results for the analytic surface indicate \pm 1 standard deviation in the state populations expected for a sample of 1500 trajectories.

these later trajectories, samples of 1500 trajectories were used to calculate the standard deviation in the state populations expected for a sample of 1500 . The comparison between the time-dependent state populations calculated on the analytic and interpolated surfaces is presented in Fig. 1.

It is clear that the relative populations of the states evaluated with the interpolated DPEM converge to the corresponding analytic surface results as the size of the data set increases. Even the interpolated DPEM using just ten data points produces a nearly quantitative description of the population dynamics. 


\section{CONCLUDING REMARKS}

A general approach to the construction of the diabatic potential energy matrix (DPEM) for multiple electronic states of polyatomic molecules has been presented. The method uses $a b$ initio quantum chemistry calculations of the adiabatic energies and derivative couplings. The DPEM is given by a modified Shepard interpolation of second order Taylor series, centered on data points scattered throughout the relevant space of molecular configurations.

While this Shepard interpolation form is formally similar to that used previously for adiabatic potential energy surfaces, the interpolation for the DPEM is complicated by three major factors: molecular coordinates which are not invariant to inversion must be used; the DPEM is not invariant to the CNPI symmetry operations as is a simple PES; and an essential factor in the DPEM, namely, the adiabatic to diabatic transformation (ADT) matrix is not provided directly by $a b$ initio quantum chemistry calculations. Methods to resolve each of these difficulties have been presented.

The approach has been tested by construction of an interpolated approximation to an analytic DPEM for $\mathrm{NH}_{3}^{+}$. The locations of data points for the interpolation have been determined by procedures very similar to those previously used to "grow" PES, except that surface hopping trajectories, rather than adiabatic trajectories, have been used to sample the relevant regions of molecular configuration space. The accuracy with which this total approach reproduces the state population dynamics on the analytic DPEM indicates that both the interpolation and data location procedures are accurate and efficient. Further testing will be required to examine how the choice of initial data set influences the rate of convergence of the interpolated DPEM, for both bound systems such as $\mathrm{NH}_{3}^{+}$and bimolecular collisions. This test case does not test how robust the procedures would be when nonremovable coupling is present. This will be the subject of future work.

The construction of single PES or DPEM by interpolation of ab initio data is a computationally intensive procedure. Experience has shown that single PES can be constructed at relatively high levels of ab initio theory for systems of several atoms. The number of data points required does increase with the dimensionality of the system, varying from around 400-1000 data points for four atoms to a few thousand data points for six to nine atoms. The construction of a DPEM will require MCSCF or MRCI ab initio calculations which are often computationally demanding, suggesting that a DPEM will be more computationally expensive than a single PES which might require MP2 or $\operatorname{CCSD}(\mathrm{T})$ calculations for sufficient accuracy. Several applications of this procedure for constructing ab initio DPEM will be necessary to determine how many data points are required for convergence.

\section{ACKNOWLEDGMENTS}

The authors wish to acknowledge the support of the Australian Research Council and the Australian Partnership for Advanced Computing. One of the authors (C.R.E.) acknowledges the support of an Australian Postgraduate Award.

\section{APPENDIX A: THE ADT MATRICES}

Here we describe how the ADT matrix is determined for all data points. In assigning the ADT matrices, we must also deal with the problem that the overall sign of the derivative coupling vector, as given by the $a b$ initio calculation, is arbitrary. The signs of the derivative couplings at all the data points must be made mutually consistent.

The basic approach to the whole task is to begin by determining the $A$ matrix at one data point and then to propagatively assign the $A$ A matrix at neighboring data points. This provides an initial estimate of the $A$ matrices. These values are then refined by optimizing the mutual consistency of all the matrices simultaneously. The details of how all this is achieved are presented here.

\section{The first matrix}

At any stage in the construction of the DPEM, we have some set of data points. To assign the $\underset{\underline{A}}{\mathrm{~A}}$ matrices, we begin with one of these points. It is convenient to choose this first data point as the data point which is closest to a conical intersection, and denote it as $\mathbf{Z}(1)$. We are free to fix the ADT matrix at one point, and to choose the sign of the derivative coupling there. So, the sign of the ab initio derivative coupling at $\mathbf{Z}(1)$ is defined to be correct. To determine $\underline{\underline{A}}(1)$, we first find the permutation of $\mathbf{Z}(1), g \circ \mathbf{Z}(1)$, that is closest to $\mathbf{Z}(1)$. In general, this is a pairwise permutation or an inversion. As $g$ is a pairwise permutation or an inversion, the midpoint $\mathbf{Z}_{\text {mid }}=\frac{1}{2}[\mathbf{Z}(1)+g \circ \mathbf{Z}(1)]$ is a point symmetric geometry and $g \circ \mathbf{Z}_{\text {mid }}=\mathbf{Z}_{\text {mid }}$.

The Taylor series for $\underset{D}{\tilde{D}}$ from $\mathbf{Z}(1)$ is calculated at the midpoint and is diagonalized,

$$
\tilde{\underline{D}}\left(\mathbf{Z}_{\text {mid }} ; 1\right)=\underline{\underline{A}}^{T}\left(\mathbf{Z}_{\text {mid }} ; 1\right) \underline{\underline{\underline{E}}}\left(\mathbf{Z}_{\text {mid }} ; 1\right) \underset{\underline{A}}{\underline{\widetilde{A}}}\left(\mathbf{Z}_{\text {mid }} ; 1\right),
$$

where $\underset{\underline{E}}{E}\left(\mathbf{Z}_{\text {mid }} ; 1\right)$ is the approximation for the adiabatic energies at $\mathbf{Z}_{\text {mid }}$ from data point 1 and $\underset{\underline{A}}{\widetilde{A}}\left(\mathbf{Z}_{\text {mid }} ; 1\right)$ is the approximation for the change in the ADT in going from data point 1 to $\mathbf{Z}_{\text {mid. }}$. Using the freedom to define the ADT matrix at a single geometry, we set $\underline{\underline{A}}(1)=\tilde{\widetilde{A}}^{T}\left(\mathbf{Z}_{\text {mid }} ; 1\right)$ so the diabatic potential at $\mathbf{Z}_{\text {mid }}$ is diagonal,

$$
\begin{aligned}
& \underline{\underline{D}}\left(\mathbf{Z}_{\text {mid }} ; 1\right)=\underline{\underline{A}}^{T}(1) \underset{\underline{D}}{\underline{D}}\left(\mathbf{Z}_{\text {mid }} ; 1\right) \underset{\underline{A}}{A}(1)
\end{aligned}
$$

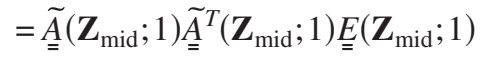

$$
\begin{aligned}
& \left.\times \underset{\underline{\widetilde{A}}}{\left(\mathbf{Z}_{\text {mid }} ; 1\right)}\right)_{\underline{\underline{A}}}^{T}\left(\mathbf{Z}_{\text {mid }} ; 1\right)=\underline{\underline{E}}\left(\mathbf{Z}_{\text {mid }} ; 1\right) \text {. }
\end{aligned}
$$

\section{Subsequent matrices}

We now choose the next data point to be assigned an $\stackrel{\underline{A}}{A}$ matrix. Suppose that the $A$ matrix has been assigned at nassign configurations (initially, nassign equals the order of the symmetry group, since only one distinct data point has been assigned). For every $\mathbf{Z}(n)$ which is not yet assigned, we evaluate 


$$
\nu_{\text {sum }}(n)=\sum_{k=1}^{\text {nssign }} \nu(Z(k), n),
$$

where $\nu(Z(k), n)$ is the primitive two-part weight function of Eq. (3.43). The data point for which $\nu_{\text {sum }}(n)$ is largest is the "closest" data point to the set of assigned data points. This is chosen to be the next data point, denoted $n$, for which $\underset{=}{A}$ is to be assigned.

The closest nneigh data points (where the ADT matrix is known) to data point $n$ are found $(\|\mathbf{Z}(n)-\mathbf{Z}(g \circ m)\|$ is smallest). When few data points have been assigned, nneigh is taken to be all the assigned data points. When many points have been assigned, nneigh is taken to be a number, e.g., 20. These neighbors of data point $n$ are labeled by the index $k$. The $k$ th neighbor is the permutation $g_{k}^{(n)}$ of data point $m_{k}^{(n)}$. The midpoint of data point $n$ and its $k$ th neighbor is

$$
\mathbf{Z}_{\text {mid }}(n, k)=\left[\mathbf{Z}(n)+\mathbf{Z}\left(g_{k}^{(n)} \circ m_{k}^{(n)}\right)\right] / 2 .
$$

We let $\underset{=}{A}(n \mid k)$ denote the value of $\underset{=}{A}(n)$ which makes $\underset{D}{D}\left(\mathbf{Z}_{\text {mid }}(k, n) ; n\right)$ consistent with $\underset{\equiv}{D}\left(\mathbf{Z}_{\text {mid }}(k, n) ; k\right)$. That is, $\stackrel{A}{A}(n \mid k)$ is the matrix which minimizes $\delta D(n, k)$,

$$
\begin{aligned}
& \delta D(n, k)=\| \underset{\equiv}{D}\left(\mathbf{Z}_{\text {mid }}(k, n) ; k\right) \\
& -\underline{A}^{T}(n \mid k) \underset{\underline{D}}{\tilde{D}}\left(\mathbf{Z}_{\text {mid }}(k, n) ; n\right) \underset{=}{A}(n \mid k) \| .
\end{aligned}
$$

If $\underset{\tilde{A}}{\widetilde{T}}\left(\mathbf{Z}_{\text {mid }}(k, n) ; n\right) \quad$ diagonalizes $\underset{D}{\widetilde{D}}\left(\mathbf{Z}_{\text {mid }}(k, n) ; n\right) \quad$ and $\underset{=}{A}\left(\mathbf{Z}_{\text {mid }}(k, n) ; k\right)$ diagonalizes $\underset{=}{D}\left(\mathbf{Z}_{\text {mid }}(k, n) ; k\right)$, then from Appendix B,

$$
\left.\underset{\underline{A}}{A}(n \mid k)=\widetilde{\underline{A}}^{T}\left(\mathbf{Z}_{\mathrm{mid}}(k, n) ; n\right)[\underline{\underline{s}} \underline{\underline{\underline{ }}}] \underline{\underline{A}} \underset{\underline{A}}{\left(\mathbf{Z}_{\mathrm{mid}}\right.}(k, n) ; k\right) .
$$

Here the correct choice of the sign change and permutation matrices $\underline{\underline{s}}$ and $\underline{\underline{p}}$ (see Appendix B) minimizes $\delta d D(k, n)$,

$$
\begin{aligned}
\delta d D(k, n)= & \| \frac{\partial}{\partial \mathbf{Z}} \underset{=}{D}\left(\mathbf{Z}_{\text {mid }}(k, n) ; k\right) \\
& -\stackrel{A}{A}^{T}(n \mid k) \frac{\partial}{\partial \mathbf{Z}} \underset{\underline{D}}{\tilde{D}}\left(\mathbf{Z}_{\text {mid }}(k, n) ; n\right) \underset{=}{A}(n \mid k) \| .
\end{aligned}
$$

In order to estimate $\underset{\equiv}{A}(n)$ based on all nneigh neighbors, we employ a weighted sum of the $\underset{=}{A}(n \mid k)$ to estimate the DPEM at data point $n$ :

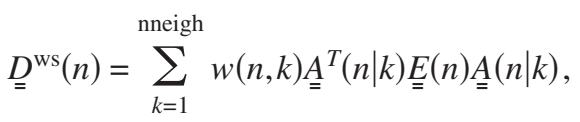

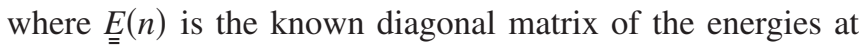
data point $n$. The weight function is described in Sec. III E. Note that the weights at $\mathbf{Z}(n)$ from data point $\mathbf{Z}(k)$ can be evaluated with the more accurate two-part weight function since the necessary confidence lengths can be evaluated without knowing the ADT matrices. The ADT matrix at data point $n$ is found by diagonalizing $D^{\mathrm{ws}}(n)$,

$$
D^{\mathrm{ws}}(n)={\underset{A}{A}}^{T}(n) \underline{\underline{E}}^{\mathrm{ws}}(n) \underset{=}{A}(n) .
$$

The greater the variance in the $\underset{A}{A}(n \mid k)$ 's, the greater the difference between $\underset{E}{E}(n)$ and $\underline{E}^{\mathrm{ws}}(n)$. This difference can be used as a gauge of the accuracy of the determination of $\underset{=}{A}(n)$. Since $\underset{=}{A}(n)$ is obtained by diagonalization, it is undetermined to within a sign change and permutation matrix, as in Eq. (B5). The correct choice for $\underline{\underline{s}}$ and $p$ is found by finding the minimum of the weighted sum of the differences in the Taylor series for the derivatives of $\underset{D}{D}$,

$$
\begin{aligned}
& \min _{\underline{\underline{s}}, \underline{\underline{\underline{p}}}} \sum_{k=1}^{\text {nneigh }} \| \frac{\partial}{\partial \mathbf{Z}} \underset{\underline{D}}{D}\left(\mathbf{Z}_{\text {mid }}(n, k) ; k\right) \\
& -\underline{\underline{A}}^{T}(n) \underline{\underline{\underline{s}}} \underline{\underline{p}}^{T} \frac{\partial}{\partial \mathbf{Z}} \underline{\underline{D}}\left(\mathbf{Z}_{\mathrm{mid}}(n, k) ; n\right) \underset{\underline{\underline{p}}}{\underline{\underline{s}}} \underline{\underline{A}}(n) \| .
\end{aligned}
$$

\section{Propagation}

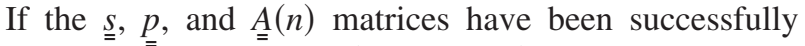
evaluated, then data point $n$ (and all $g \circ n$ ) is added to the set of assigned data points, and we return to Appendix A 2 above to choose the next point to be assigned. However, it may be that the choice of correct $\underline{\underline{s}}$ and $\underline{\underline{p}}$ in Eq. (A10) is ambiguous. That is, more than one choice of $\underline{\underline{s}}$ and $p$ yield a small norm in (A10). This can occur if the data points are sparsely distributed in the region near data point $n$, and/or the derivative coupling is small in this region (so that the nonremovable component is relatively large). In this case, $\underset{=}{A}(n)$ is not assigned. We return to Appendix A 2 and choose the next closest point to the current assigned set to be the next point to be assigned. In the exhaustive propagation of this iteration, data point $n$ will be considered again for assignment at some later iteration. At that time, more $A$ matrices will have been assigned, so that $\underset{\equiv}{A}(n)$ may then be successfully assigned. Eventually, an estimate of the $\underset{\underline{A}}{A}$ for every data point will be obtained.

\section{Refinement of the matrices}

Here we discuss how disagreements between the Taylor series for $\underset{D}{D}$ can be used to further refine the $\underset{\equiv}{A}(n)$. We start with the $\underset{A}{A}(n)$ values determined by Eq. (A9). All the Taylor

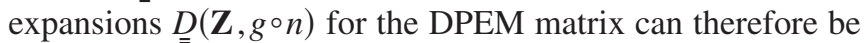
evaluated. The disagreement between the Taylor series for the diabatic potential, from data point $n$ and its $k$ th neighbor to their midpoint, is given by

$$
\operatorname{error}(n, k)=\left\|\underset{=}{D}\left[\mathbf{Z}_{\text {mid }}(n, k) ; n\right]-\underset{=}{D}\left[\mathbf{Z}_{\text {mid }}(n, k) ; k\right]\right\|^{2} .
$$

These disagreements, or errors, are combined in a weighted sum

$$
\text { error }=\sum_{n=1}^{\text {ndata nneigh }} \sum_{k=1} w(n, k) \operatorname{error}(n, k),
$$

where $w(n, k)$ uses the two-part weight function of Eq. (3.43). In an important modification of subsequent matrices above, the nneigh neighbors can include permutations, $g \circ n$, of the data point itself. As we show below, inclusion of these permutation-inversions in the set of neighbors imposes boundary conditions on the ADT matrix. We minimize the error by varying all the $A(n)$.

Aside. In place of Eq. (A11), it would also be possible to consider the disagreement in the gradient of $\underset{\underline{D}}{\text { between }}$ neighboring data points: 


$$
\operatorname{derror}(n, k)=\left\|\nabla \underset{D}{D}\left[\mathbf{Z}_{\text {mid }}(n, k) ; n\right]-\nabla \underset{\equiv}{D}\left[\mathbf{Z}_{\text {mid }}(n, k) ; k\right]\right\|^{2} .
$$

The sum of these disagreements over all neighbors and data points in then given by

$$
\text { derror }=\sum_{n=1}^{\text {ndata nneigh }} \sum_{k=1} w(n, k) \text { derror }(n, k) .
$$

We might then optimize the $\underset{\underline{A}}{A}(n)$ by minimizing derror.

Whether we minimize the value of error or derror, we need to vary the $A(n)$ matrices while retaining the orthogonality property of each $A(n)$. In Appendix $\mathrm{C}$ we show how the local changes in $A(n)$ can be parametrized by a set of angles $\theta_{a, b}^{(n)}$, where $1 \leqslant a<b \leqslant$ nstates. For nstates $\geqslant 3$, it is not possible to globally parametrize the orthogonal matrices: for a given parameterization of the orthogonal matrices, there exist matrices for which the derivative of the ADT matrix with respect to one of the parameters is singular. Mathematically, this is the same problem encountered in describing the internal coordinates of a molecule. To integrate the derivative couplings, Vertesi et al. have used a global parametrization for the ADT matrix. ${ }^{27}$ This parametrization leads to complicated coupled equations relating the parameters and exhibits singularities in the parameters that have to be carefully treated. Appendix D derives the derivative of error with respect to all the angles $\theta_{a, b}^{(n)}$ for the local parametrization for the ADT matrix. Starting with an estimate of $\underset{\underline{A}}{(n)}(n)$, error is minimized using the conjugate gradient algorithm (see Ref. 26, p. 301) to obtain an optimal set of $\underset{\underline{A}}{(}(n)$.

\section{Boundary conditions on $\underline{\underline{A}(n)}$}

In Appendix A 4 above, the nneigh neighbors of data point $n$ include permutations of data point $n$. Although the final optimal ADT matrices are unknown at these points, the permutations of $n$ impose boundary conditions on the refinement procedure for the diabatic potential. In this section we show how the permutations of data point $n$ impose boundary conditions on $A(n)$.

If the $k$ th neighbor of data point $n$ is a pairwise permutation or the inversion of data point $n$, the midpoint

$$
\mathbf{Z}_{\text {mid }}(n, k)=\left[\mathbf{Z}(n)+\mathbf{Z}\left(g_{k}^{(n)} \circ n\right)\right] / 2
$$

is a point symmetric geometry, as $g_{k}^{(n)} g_{k}^{(n)}=I$. The geometry $\mathbf{Z}_{\text {mid }}(n, k)$ possesses at least a plane of symmetry. This is a comparatively common geometrical feature in chemical systems. If, for example, the corresponding symmetry matrix is an improper rotation, then some elements of the diabatic potential are zero. In general, if the $k$ th neighbor of data point $n$ is the permutation $g_{k}^{(n)}$ of data point $n$, we have

$$
\underline{\underline{D}}\left(\mathbf{Z}_{\text {mid }}(n, k)\right)=g_{k}^{(n)} \circ \underline{\underline{D}}\left(\mathbf{Z}_{\text {mid }}(n, k)\right) .
$$

Therefore,

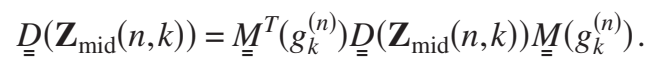

Equation (A17) imposes a constraint, or boundary condition, on the DPEM at a geometry which has point-group symmetry. In addition, there are constraints on the derivatives of the diabatic potential at symmetric geometries,

$$
\frac{\partial}{\partial \mathbf{Z}_{l}} \stackrel{D}{ }\left(\mathbf{Z}_{\text {mid }}(n, k)\right)=g_{k}^{(n)} \circ \frac{\partial}{\partial \mathbf{Z}_{l}} \stackrel{D}{=}\left(\mathbf{Z}_{\text {mid }}(n, k)\right),
$$

$$
\therefore \frac{\partial}{\partial \mathbf{Z}_{l}} \underline{\underline{D}}\left(\mathbf{Z}_{\text {mid }}(n, k)\right)=\underline{\underline{M}}^{T}\left(g_{k}^{(n)}\right) \frac{\partial}{\partial Z_{g_{k}(n)_{o}}} \underline{D}\left(\mathbf{Z}_{\text {mid }}(n, k)\right) \underline{\underline{M}}\left(g_{k}^{(n)}\right) \text {. }
$$

The inclusion of permutation-inversions of the data point itself $(g \circ n)$ in Eq. (A12) imposes these boundary conditions on $\underset{D}{D}$ on the optimization of the ADT matrices.

\section{APPENDIX B: MATRIX SIMILARITY}

For $N \times N$ symmetric matrices $\underline{\underline{B}}$ and $\underline{\underline{C}}$, we want to find the orthogonal transformation $\underline{\underline{U}}$, which minimizes $\delta$ (the Frobenius norm),

$$
\delta=\left\|\underline{\underline{C}}-\underline{\underline{U}}^{T} \underline{\underline{B}} \underline{\underline{U}}\right\|^{2} .
$$

Let

$$
\underline{\underline{B}}=\underline{\underline{V}}^{T} \underline{\underline{\lambda}}^{B} \underline{\underline{V}}, \quad \underline{\underline{C}}=\underline{\underline{W}}^{T} \underline{\underline{\lambda}}^{C} \underline{\underline{W}},
$$

where $\underline{\underline{V}}$ and $\underline{\underline{W}}$ are orthogonal matrices and $\underline{\underline{\lambda}}^{B}$ and $\underline{\underline{\lambda}}^{C}$ are diagonal matrices of eigenvalues. Substituting

$$
\underline{\underline{U}}=\underline{\underline{V}}^{T} \underline{\underline{W}}
$$

into Eq. (B1) gives

$$
\begin{aligned}
\delta & =\left\|\underline{\underline{U}}^{T} \underline{\underline{B}} \underline{\underline{U}}-\underline{\underline{C}}\right\|^{2}=\left\|\underline{\underline{W}}^{T} \underline{\underline{V}} \underline{\underline{B}} \underline{\underline{V}}^{T} \underline{\underline{W}}-\underline{\underline{C}}\right\|^{2}=\left\|\underline{\underline{W}}^{T} \underline{\underline{\lambda^{B}}}{ }^{W} \underline{\underline{W}}-\underline{\underline{C}}\right\|^{2} \\
& =\left\|\underline{\underline{W}}^{T}\left(\underline{\underline{\underline{\lambda}}}^{B}-\underline{\underline{\lambda}}^{C}\right) \underline{\underline{W}}\right\|^{2}=\left\|\underline{\underline{\lambda}}^{B}-\underline{\underline{\lambda}}^{C}\right\|^{2} .
\end{aligned}
$$

The last line follows as the norm is invariant to orthogonal transformations. Hence, the closer the eigenvalues of $\underline{\underline{B}}$ and $\underline{\underline{C}}$ are, the smaller the difference in Eq. (B4) is, and if $\underline{\underline{B}}$ and $\underline{\underline{C}}$ have the same eigenvalues, (B3) gives $\delta=0$. Conversely, defining $U$ by (4.3) yields a value for $\delta$ which depends on the square of the difference in eigenvalues and vanishes when $\underline{\underline{B}}$ and $\underline{\underline{C}}$ have the same eigenvalues. We therefore adopt (B3) to define a suitable orthogonal transformation which minimizes $\delta$.

It is important to note that when any matrix is diagonalized, the sign of the eigenvectors and the ordering of the eigenvalues and eigenvectors are uncertain. Let $p$ be an element of $P$, the group of $N \times N$ permutation matrices, and let $\underline{\underline{s}}$ be a sign change matrix, one of the groups $S$ of diagonal matrices with elements of \pm 1 .

The uncertainty in signs and ordering is reflected in the definition of $\underline{\underline{U}}$,

$$
\underline{\underline{U}}=\underline{\underline{V}}^{T}[\underline{\underline{s}} \underline{\underline{p}}] \underline{\underline{W}} .
$$

From the above analysis, each of these solutions for $\underline{\underline{U} \text {, when }}$ substituted into Eq. (B1) is stationary to perturbations of $\underline{\underline{U}}$. The correct choice of permutation and sign corresponds to the smallest value of $\delta$. Unfortunately, as $\underline{\underline{U}}^{T} \underline{\underline{B}} \underline{\underline{U}}$ is invariant to sign changes, we cannot use Eq. (B1) to determine the matrix $\underline{\underline{s}}$,

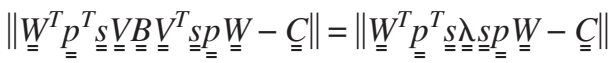

$$
\begin{aligned}
& =\left\|\underline{\underline{W}}^{T} \underline{\underline{p}}^{T} \underline{\underline{\lambda}} \underline{\underline{p}} \underline{\underline{W}}-\underline{\underline{C}}\right\| .
\end{aligned}
$$

However, the matrix $\nabla \underline{\underline{B}}$ is not diagonalized by $\underline{\underline{V}}^{T}$, so we 
determine $\underline{\underline{p}}$ and $\underline{\underline{\underline{S}}}$ by finding $\underline{\underline{p}}$ and $\underline{\underline{\underline{S}}}$ which give

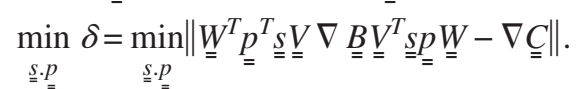

For different choices of $\underline{p}$ and $\underline{\underline{s}}$, the ratio of the smallest $\delta$ value to the next smallest $\delta$ value can be used to monitor how sensitive the assignment is. Furthermore, Eq. (B1) is unchanged by the overall sign of $\underline{\underline{U}}$. This means that the number of sign change matrices that need to be considered can be halved by taking the 1,1 element of all the $\underline{\underline{s}}$ matrices to be +1 .

\section{APPENDIX C: VARIATIONS OF ORTHOGONAL MATRICES}

To minimize error $(n)$ in (A12) or derror( $n)$ in (A14), we first need to discuss how to change the $\underset{\underline{A}}{(}(n)$ matrix and ensure that it is still orthogonal.

A finite change in the nstates $\times$ nstates orthogonal matrix $\stackrel{A}{A}$ can be parametrized by nstates(nstates- 1 ) $/ 2$ angles $\theta_{i, j}$ where $1 \leqslant i<j \leqslant$ nstates. $\theta_{i, j}$ is the angle through which the $\{i, i\},\{i, j\},\{j, i\}$, and $\{j, j\}$ elements of $A$ are rotated between one another by the matrix $R\left(i, j ; \theta_{i, j}\right)$. The elements of the (unitary) matrix $\underline{\underline{R}}\left(i, j ; \theta_{i, j}\right)$ are

$$
\begin{aligned}
{\left[\underline{\underline{R}}\left(i, j ; \theta_{i, j}\right)\right]_{k, l}=} & \delta_{k, l}+\delta_{k, i} \delta_{l, i}\left(\cos \theta_{i, j}-1\right)+\delta_{k, i} \delta_{l, j} \sin \theta_{i, j} \\
& -\delta_{k, j} \delta_{l, i} \sin \theta_{i, j}+\delta_{k, j} \delta_{l, j}\left(\cos \theta_{l, j}-1\right) .
\end{aligned}
$$

This is the finite rotation generated by the infinitesimal generator $\underline{\underline{\rho}}(i, j)$,

$$
[\underline{\underline{\rho}}(i, j)]_{a, b}=\delta_{a, i} \delta_{b, j}-\delta_{a, j} \delta_{b, i} .
$$

One can show that

$$
\exp \left[\theta_{i, j} \underline{\underline{\rho}}\right]=\underline{\underline{R}}\left(i, j ; \theta_{i, j}\right) .
$$

If $\theta_{i, j}=0$, then $\underline{\underline{R}}(i, j ; 0)=\underline{\underline{\mathbf{I}}}$, the unit matrix. We define a new matrix, $\widetilde{A}$,

$$
\tilde{\underline{A}}=\underset{\underline{A}}{A} \prod_{(i, j)} \underline{\underline{R}}\left(i, j ; \theta_{i, j}\right) .
$$

It is a simple matter to show that $\underset{\tilde{A}}{\tilde{A}}$ is unitary. If all the angles $\theta_{i, j}$ are zero, then $\underset{\underline{A}}{\tilde{A}}=\underline{\underline{A}}$, or else $\underline{\overline{\widetilde{A}}}$ is a finite variation of $\underline{\underline{A}}$. The derivative of $\tilde{\overline{\tilde{A}}}$ with respect to $\theta_{a, b}$ is

$$
\begin{aligned}
& \frac{\partial}{\partial \theta_{a, b}} \underset{\underline{A}}{\tilde{A}}=\underset{=}{A} \frac{\partial}{\partial \theta_{a, b}} \prod_{(i, j)} \underset{=}{R}\left(i, j ; \theta_{i, j}\right) \\
& =\underset{\underline{(i, j)<(a, b)}}{A} \prod_{\underline{R}}\left(i, j ; \theta_{i, j}\right) \frac{\partial}{\partial \theta_{a, b}} \underline{R}\left(a, b ; \theta_{a, b}\right) \\
& \times \prod_{(i, j)>(a, b)} \underset{=}{R}\left(i, j ; \theta_{i, j}\right) \\
& =\stackrel{A}{=} \prod_{(i, j)<(a, b)} \underset{=}{R}\left(i, j ; \theta_{i, j}\right) Q\left(a, b ; \theta_{a, b}\right) \\
& \times \prod_{(i, j)>(a, b)} \underset{=}{R}\left(i, j ; \theta_{i, j}\right),
\end{aligned}
$$

where

$$
\begin{aligned}
{\left[Q\left(a, b ; \theta_{a, b}\right)\right]_{k, l}=} & -\delta_{k, a} \delta_{l, a} \sin \theta_{a, b}+\delta_{k, a} \delta_{l, b} \cos \theta_{a, b} \\
& -\delta_{k, b} \delta_{l, a} \cos \theta_{a, b}-\delta_{k, b} \delta_{l, b} \sin \theta_{a, b} .
\end{aligned}
$$

The strength of this approach is that $\underline{\underline{A}}$ is not globally parameterized; however, the parameterization of variations to $\underset{\underline{A}}{A}$ in terms of $\tilde{A}$ is valid for small finite variations.

\section{APPENDIX D: DERIVATIVES OF ERROR}

The disagreement between the Taylor series for the diabatic potential, from data point $n$ and its $k$ th neighbor to their midpoint, is given by [see Eqs. (A11) and (A12)]

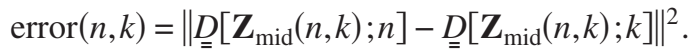

These disagreements, or errors, are combined in a weighted sum over all neighbors of all data points and are denoted as

$$
\text { error }=\sum_{n=1}^{\text {ndata }} \sum_{k=1}^{\text {nneigh }} w(n, k) \text { error }(n, k) \text {, }
$$

where $w(n, k)$ is the two-part weight function of Eqs. (3.39) and (3.43). The ADT matrices at each data point, $A(n)$, determine the value of error, and the most consistent set of ADT matrices is that which minimizes the value of error. Beginning with the set of ADT matrices determined by the propagative assignment procedure (see Appendix A 3), all the $A(n)$ matrices are varied by varying the angles $\theta_{i, j}$ of Appendix $\mathrm{C}$, using a conjugate gradient method, to minimize error. To implement the conjugate gradient method, we must have explicit expressions for the derivatives of each $A(n)$ with respect to the associated angles $\theta_{i, j}$ (see Appendix C).

First we write error explicitly as

$$
\begin{aligned}
\text { error }= & \sum_{n=1}^{\text {ndata nneigh }} \sum_{k=1} w(n, k) \| \underline{\underline{D}}\left[\mathbf{Z}_{\text {mid }}(n, k) ; n\right] \\
& -\underset{\underline{D}}{D}\left[\mathbf{Z}_{\text {mid }}(n, k) ; k\right] \|^{2} \\
= & \sum_{n=1}^{\text {ndata nneigh }} \sum_{k=1}^{\text {nstates nstates }} w(n, k) \sum_{i=1} \sum_{j=1} \operatorname{error}(n, k, i, j)^{2},
\end{aligned}
$$

where

$$
\begin{aligned}
\operatorname{error}(n, k, i, j) & =\left\{\underline{\underline{A}}^{T}(n) \underset{\underline{D}}{\underline{\underline{D}}}\left[\mathbf{Z}_{\text {mid }}(n, k) ; n\right] \underline{\underline{A}}(n)-\underline{\underline{A}}^{T}\left(g^{(k)}\right.\right. \\
& \left.\left.\circ m^{(k)}\right) \tilde{\underline{D}}\left[\mathbf{Z}_{\text {mid }}(n, k) ; g^{(k)} \circ m^{(k)}\right] \underline{\underline{A}}\left(g^{(k)} \circ m^{(k)}\right)\right\}_{i, j},
\end{aligned}
$$

and the $k$ th neighbor is some permutation-inversion of data point $m, g^{(k)} \circ m^{(k)}$. Using the symmetry properties of the ADT matrices [see Eq. (3.23)], 


$$
\begin{aligned}
\operatorname{error}(n, k, i, j) & =\left\{\underline{\underline{A}}^{T}(n) \underset{\underline{D}}{\underline{D}}\left[\mathbf{Z}_{\mathrm{mid}}(n, k) ; n\right] \underline{\underline{A}}(n)-\underline{\underline{M}}^{T}\left(g^{(k)}\right) \underline{\underline{A^{T}}}\left(m^{(k)}\right) \underline{\underline{S}}\left(g^{(k)}\right) \underline{\underline{D}}\left[\mathbf{Z}_{\mathrm{mid}}(n, k) ; g^{(k)} \circ m^{(k)}\right] \underline{\underline{S}}\left(g^{(k)}\right) \underline{\underline{A}}\left(m^{(k)}\right) \underline{\underline{M}}\left(g^{(k)}\right)\right\}_{i, j} \\
& =\left\{\underline{\underline{A}}^{T}(n) \underline{\underline{P}}(n) \underline{\underline{A}}(n)-\underline{\underline{M}}^{T}\left(g^{(k)}\right) \underline{\underline{A}}^{T}\left(m^{(k)}\right) \underline{\underline{P}}(k) \underline{\underline{A}}\left(m^{(k)}\right) \underline{\underline{M}}\left(g^{(k)}\right)\right\}_{i, j} .
\end{aligned}
$$

Here we have introduced two new matrices to abbreviate the notation. The $\underline{P}(n)$ and $\underline{\underline{P}}(k)$ matrices are independent of the ADT matrices, and are therefore effective constants in this context.

Now we replace each ADT matrix $\underline{\underline{A}(n)}$ with the variable matrix $\underset{\underline{A}}{\widetilde{A}}(n)$ introduced in Appendix C, to write

$$
\begin{aligned}
\operatorname{error}(n, k, i, j)= & \left\{\underline{\underline{\tilde{A}}}^{T}(n) \underline{\underline{P}}(n)\right) \\
& \left.-\underline{\underline{M}}^{T}\left(g^{(k)}\right) \underline{\underline{\underline{A}}}(n)\left(m^{T}\right) \underline{\underline{\underline{P}}}(k) \underline{\underline{A}}\left(m^{(k)}\right) \underline{\underline{M}}\left(g^{(k)}\right)\right\}_{i, j} .
\end{aligned}
$$

determine the variation in the ADT matrix for data point $p$ is given by

$$
\begin{aligned}
& \frac{\partial \text { error }}{\partial \theta_{a, b}^{(p)}}=2 \sum_{n=1}^{\text {ndata nneigh }} \sum_{k=1} w(n, k) \\
& \times \sum_{i=1}^{\text {nstates }} \sum_{j=1}^{\text {nstates }} \operatorname{error}(n, k, i, j) \frac{\partial}{\partial \theta_{a, b}^{(p)}} \operatorname{error}(n, k, i, j),
\end{aligned}
$$

where

Then the derivative of error with respect to the angles which

$$
\begin{aligned}
\frac{\partial}{\partial \theta_{a, b}^{(p)}} \operatorname{error}(n, k, i, j)= & \left\{\delta_{p, n}\left[\frac{\partial \underline{\underline{\underline{A}}}^{T}}{\partial \theta_{a, b}^{(p)}} \underline{\underline{P}}(n) \underline{\underline{A}}(n)+\underline{\underline{A}}^{T}(n) \underline{\underline{P}}(n) \frac{\partial \underline{\underline{\tilde{A}}}(n)}{\partial \theta_{a, b}^{(p)}}\right]-\delta_{p, m^{(k)}}\right. \\
& \left.\times\left[\underline{\underline{M}}^{T}\left(g^{(k)}\right) \frac{\partial \underline{\underline{\tilde{A}^{T}}}\left(m^{(k)}\right)}{\partial \theta_{a, b}^{(p)}} \underline{\underline{P}}(k) \underline{\underline{A}}(n) \underline{\underline{M}}\left(g^{(k)}\right)+\underline{\underline{M}}^{T}\left(g^{(k)}\right) \underline{\underline{A}}^{T}\left(m^{(k)}\right) \underline{\underline{P}}(k) \frac{\partial \widetilde{\underline{A}}\left(m^{(k)}\right)}{\partial \theta_{a, b}^{(p)}} \underline{\underline{M}}\left(g^{(k)}\right)\right]\right\}_{i, j}
\end{aligned}
$$

The derivative of the $\widetilde{A}$ matrices with respect to the associated angles are given in Eqs. (C5) and (C6).

\section{APPENDIX E: CONFIDENCE LENGTHS}

The confidence lengths used in interpolation of a PES are evaluated from a consideration of the errors in a Taylor series for the gradient of the PES. These errors can be evaluated at neighboring data points, since the PES gradients are known at these locations from ab initio calculations. For interpolation of the DPEM, the confidence lengths are evaluated from the errors in the Taylor series for the DPEM. To

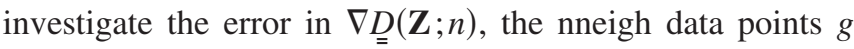
o $m$ closest to data point $n$ are found from the set of permutations of the data points (excluding data point $n$ ). As in Eq. (3.34), we define the midpoint $\mathbf{Z}_{\text {mid }}$ between data points $\mathrm{n}$ and $g \circ \mathrm{m}$. Now,

$$
\underset{\underline{D}}{D}\left(\mathbf{Z}_{\mathrm{mid}} ; n\right) \approx \underset{\underline{D}}{D}\left(\mathbf{Z}_{\mathrm{mid}} ; g \circ m\right)
$$

implies

$$
\widetilde{\underline{D}}\left(\mathbf{Z}_{\text {mid }} ; n\right) \approx \underline{\underline{W}}^{T} \underline{\underline{D}}\left(\mathbf{Z}_{\text {mid }} ; g \circ m\right) \underline{\underline{W}},
$$

where $W$ is a unitary matrix which can be found as follows. First we diagonalize $\underset{\underline{D}}{\tilde{D}}\left(\mathbf{Z}_{\text {mid }} ; n\right)$ and $\underset{\underline{D}}{\tilde{D}}\left(\mathbf{Z}_{\text {mid }} ; g \circ m\right)$,

$$
\begin{aligned}
& \widetilde{\underline{D}}\left(\mathbf{Z}_{\mathrm{mid}} ; n\right)=\underline{\underline{P}}^{T} \underline{\underline{\Lambda}}(n) \underline{\underline{P}}, \\
& \widetilde{\underline{D}}\left(\mathbf{Z}_{\text {mid }} ; g \circ m\right)=\underline{\underline{Q}}^{T} \Lambda(g \circ m) \underline{\underline{Q}} .
\end{aligned}
$$

Then setting

$$
\underline{\underline{P}}^{T} \underline{\underline{\Lambda}}(n) \underline{\underline{P}}=\underline{\underline{W}} \underline{\underline{Q}}^{T} \underline{\underline{\Lambda}}(\mathrm{g} \circ \mathrm{m}) \underline{\underline{\underline{W}}} \underline{\underline{W}}
$$

gives

$$
\underline{\underline{W}}=\underline{\underline{Q}}^{T} \underline{\underline{P}},
$$

subject to determining the signs and order of the eigenvectors, as in Appendix B. The matrix $\underline{\underline{W}}$ has been evaluated only from a knowledge of $\widetilde{D}\left(\mathbf{Z}_{\text {mid }} ; n\right)$ and $\widetilde{D}\left(\mathbf{Z}_{\text {mid }} ; g \circ m\right)$, so it does not depend on the unknown ADT matrices $A(n)$ and $\underline{\underline{A}}(\mathrm{~g} \circ \mathrm{m})$. Since the unitary matrix $W$ is known, we can evaluate the difference $\delta \tilde{\underline{D}}$,

$$
\delta \tilde{\underline{D}}=\tilde{\underline{D}}\left(\mathbf{Z}_{\text {mid }} ; n\right)-\underline{\underline{W}}^{T} \underline{\underline{D}}\left(\mathbf{Z}_{\text {mid }} ; g \circ m\right) \underline{\underline{W}} .
$$

If the coefficients in the second order Taylor series were exact, then the difference or error $\delta \tilde{D}$ is proportional to the cube of the displacement between the data points,

$$
\delta \tilde{\underline{D}} \sim \mathrm{O}\left(\|\mathbf{Z}(n)-g \circ \mathbf{Z}(m)\|^{3}\right) .
$$

Moreover, for each individual coordinate, $Z_{l}$, we have an 
error given by

$$
\begin{aligned}
& {\left[Z_{l}(n)-Z_{l}(g \circ m)\right]} \\
& \quad \times\left[\frac{\partial}{\partial Z_{l}} \tilde{D}(\mathbf{Z} ; n)-\underline{W}^{T} \frac{\partial}{\partial Z_{l}} \tilde{D}(\mathbf{Z} ; g \circ m) \underset{=}{W}\right]_{\mathbf{Z}=\mathbf{Z} \operatorname{mid}} \\
& \quad \sim \mathrm{O}\left(\|\mathbf{Z}(n)-g \circ \mathbf{Z}(m)\|^{3}\right) .
\end{aligned}
$$

In (E8), the derivatives are not true partial derivatives when the number of primitive coordinates exceeds $3 N-6$. In that case, the symbols refer to the apparent dependence of the
Taylor series on $Z_{i}$ through the definition of the independent coordinates of Eq. (3.6).

If the closest data points, $\mathbf{Z}(\mathrm{g} \circ \mathrm{m})$, are randomly distributed about $\mathbf{Z}(n)$, we might expect that the left-hand side of (E8) might average to zero for a set of such neighbors, since positive and negative errors are equally likely. Assuming that these errors in (E8) are normally distributed, a Bayesian analysis ${ }^{19}$ provides an estimate of the confidence length within which the absolute values of these errors are below a given tolerance,

$$
d_{l}(n)=\left\{\sum_{k=1}^{\text {nneigh }} \frac{\left[Z_{l}(n)-Z_{l}(k)\right]^{2}\left[\left(\partial / \partial Z_{l}\right) \widetilde{\underline{D}}(\mathbf{Z} ; n)-W(\underline{\underline{n}})^{T}\left(\partial / \partial Z_{l}\right) \underline{\underline{\underline{D}}}(\mathbf{Z} ; k) W(k)\right]_{\mathbf{Z}=\mathbf{Z}_{\text {mid }}^{(k)}}^{2}}{\text { nneigh }\left\|\mathbf{Z}_{\text {mid }}^{(k)}-\mathbf{Z}(n)\right\|^{6} E_{\text {tol }}^{2}} .\right.
$$

The number of neighboring data points considered for PES confidence lengths has varied with the dimensionality of the system. In the $\mathrm{NH}_{3}$ system considered here, nneigh $=20$. The energy tolerance $E_{\text {tot }}$ is taken to be $0.2 \mathrm{mH}$ a value typically used for interpolated PES.

\section{APPENDIX F: SYMMETRY MATRICES}

The DPEM transforms nontrivially on permutation of identical atoms or under inversion as shown in Eq. (3.24). The symmetry matrices $\underset{\underline{M}}{M}(g)$ can be determined by inspection of the electronic wave functions at point symmetric geometries. However, it is preferable to have an automated procedure for determining the symmetry matricesespecially in high dimensional systems where an exhaustive characterization of the potential energy surfaces is prohibitive.

The first element of the symmetry group, denoted $g_{1}$, is taken to be the identity operation. The corresponding symmetry matrix is the identity. The automated procedure for determining the remaining $\underline{\underline{M}}(\mathrm{~g})$ matrices is carried out at the same time as the ADT matrices are assigned. The first ADT matrix, $\underset{=}{A}(1)$, is determined at data point $\mathbf{Z}(1)$ [see Eqs. (A1) and (A2)]. We find the permutation $g$ which gives $g \circ \mathbf{Z}(1)$ as close as possible to $\mathbf{Z}(1)$, and define their midpoint, $\mathbf{Z}_{\text {mid }}$ $=\frac{1}{2}[\mathbf{Z}(1)+g \circ \mathbf{Z}(1)]$. The permutation $g$ must be a pairwise permutation or the inversion operation. We take $g_{2}=g$, and note that $g_{2} \circ \mathbf{Z}_{\text {mid }}=\mathbf{Z}_{\text {mid }}$. The first ADT matrix, $\underset{=}{A}(1)$, is fixed by (arbitrarily) setting [see Eq. (A2)]

$$
\underset{\underline{D}}{D}\left(\mathbf{Z}_{\text {mid }} ; 1\right)=\underline{\underline{E}}\left(\mathbf{Z}_{\text {mid }} ; 1\right) \text {. }
$$

We then compare the Taylor series for the diabatic potential from $\mathbf{Z}(1)$ and $g_{2} \circ \mathbf{Z}(1)$ at their midpoint $\mathbf{Z}_{\text {mid }}$. The Taylor series for the diabatic potential from data point 1 is given by (F1). As $g_{2} \circ \mathbf{Z}_{\text {mid }}=\mathbf{Z}_{\text {mid }}$ and $\underset{\equiv}{D}\left(\mathbf{Z}_{\text {mid }} ; 1\right)$ is diagonal at $\mathbf{Z}_{\text {mid }}$, the Taylor series from data point $g_{2} \circ 1$ is

$$
\begin{aligned}
\underline{\underline{D}}\left(\mathbf{Z}_{\text {mid }} ; g_{2} \circ 1\right) & =\underline{\underline{M}}^{T}\left(g_{2}\right) \underline{\underline{A}}^{T}(1) \underline{\underline{\underline{D}}}\left(g_{2}^{-1} \circ \mathbf{Z}_{\text {mid }} ; 1\right) \underline{\underline{A}}(1) \underline{\underline{M}}\left(g_{2}\right) \\
& =\underline{\underline{M}}^{T}\left(g_{2}\right) \underline{\underline{A^{T}}}(1) \underline{\underline{\underline{D}}}\left(\mathbf{Z}_{\text {mid }} ; 1\right) \underline{\underline{A}}(1) \underline{\underline{M}}\left(g_{2}\right) \\
& =\underline{\underline{M}}^{T}\left(g_{2}\right) \underline{\underline{D}}\left(\mathbf{Z}_{\text {mid }} ; 1\right) \underline{\underline{M}}\left(g_{2}\right) \\
& =\underline{\underline{M}}^{T}\left(g_{2}\right) \underline{\underline{E}}\left(\mathbf{Z}_{\text {mid }} ; 1\right) \underline{\underline{M}}\left(g_{2}\right) .
\end{aligned}
$$

The Taylor series from $\mathbf{Z}(1)$ and $g_{2} \circ \mathbf{Z}(1)$ should agree closely; that is,

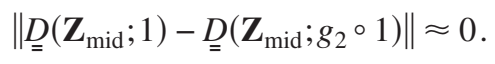

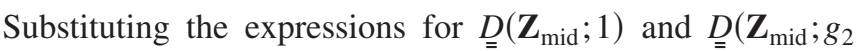
$\circ 1)$ gives

$$
\left\|\underline{\underline{E}}\left(\mathbf{Z}_{\mathrm{mid}} ; 1\right)-\underline{\underline{M}}^{T}\left(g_{2}\right) \underline{\underline{E}}\left(\mathbf{Z}_{\mathrm{mid}} ; 1\right) \underline{\underline{M}}\left(g_{2}\right)\right\| \approx 0
$$

If $M\left(g_{2}\right)$ is one of the "sign change" matrices (the set of diagonal matrices with elements of \pm 1 ), then the left-hand side of (F4) is exactly zero. Any other choice for $\underline{\underline{M}}\left(g_{2}\right)$ will result in the left-hand side of (F5) being nonzero.

The correct choice of the sign change matrix for $\underline{\underline{M}}\left(g_{2}\right)$ is the one that minimizes the disagreement between the Taylor series of derivatives of $\underset{D}{D}$ from $\mathbf{Z}(1)$ and $g_{2} \circ \mathbf{Z}(1)$ at $\mathbf{Z}_{\text {mid }}$.

$$
\min _{\underline{\underline{M}}\left(g_{2}\right) \in S}\left\|\frac{\partial}{\partial \mathbf{Z}} \underset{=}{D}\left(\mathbf{Z}_{\text {mid }} ; 1\right)-\frac{\partial}{\partial \mathbf{Z}} \underset{=}{D}\left(\mathbf{Z}_{\text {mid }} ; g_{2} \circ 1\right)\right\| .
$$

$\underline{\underline{M}}\left(g_{2}\right)$ is the sign change matrix that results in the smallest value of (F5).

Now that the second symmetry matrix has been determined, it is possible to assign the remaining matrices consecutively, as follows. Suppose we have the symmetry matrices determined for permutations $g_{1}$ through $g_{n}$, consecutively. The undetermined permutation $g$ for which $g \circ \mathbf{Z}(1)$ is closest to $g_{i} \circ \mathbf{Z}(1)$, for any $i<n$, is assigned next. This permutation is denoted as $g_{n+1}$. The Taylor series for 
the diabatic potentials are compared at the midpoints of $g_{i}$ $\circ \mathbf{Z}(1)$ and $g_{n+1} \circ \mathbf{Z}(1)$, denoted again as $\mathbf{Z}_{\text {mid }}$,

$\underset{\underline{D}}{D}\left(\mathbf{Z}_{\text {mid }} ; g_{i} \circ 1\right)=\underline{\underline{M}}^{T}\left(g_{i}\right){\underset{A}{A}}^{T}(1) \underset{\underline{D}}{\tilde{D}}\left(\mathbf{Z}_{\text {mid }} ; 1\right) \underset{=}{A}(1) \underset{=}{M}\left(g_{i}\right)$,

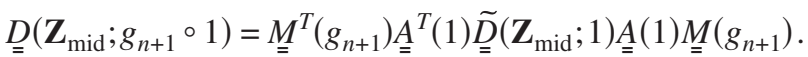

The matrix $M\left(g_{n+1}\right)$ is found by applying the assignment procedure outlined in Appendix B to

$$
\begin{aligned}
\left\|\underset{\underline{D}}{D}\left(\mathbf{Z}_{\text {mid }} ; g_{i} \circ 1\right)-\underset{\underline{D}}{D}\left(\mathbf{Z}_{\text {mid }} ; g_{n+1} \circ 1\right)\right\| \\
=\| \underline{\underline{D}}\left(\mathbf{Z}_{\text {mid }} ; g_{i} \circ 1\right)-\underline{\underline{M}}^{T}\left(g_{n+1}\right) \\
\quad \times\left[\underline{\underline{A}}^{T}(1) \underset{\underline{D}}{\widetilde{D}}\left(\mathbf{Z}_{\text {mid }} ; g_{n+1} \circ 1\right) \underset{\underline{A}}{A}(1)\right] \underline{\underline{M}}\left(g_{n+1}\right) \| .
\end{aligned}
$$

To summarize, $M\left(g_{n+1}\right)$ is constructed by diagonalizing

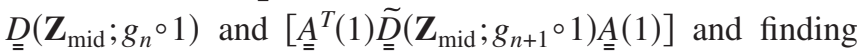
which choice of sign change and permutation matrix minimizes the disagreement between the Taylor series for the derivatives of $\underset{D}{D}$ at $\mathbf{Z}_{\text {mid }}$.

Thus, from $\underline{\underline{M}}\left(g_{1}\right)$ and $\underline{\underline{M}}\left(g_{2}\right)$, we evaluate $\underline{\underline{M}}\left(g_{3}\right)$, and so on, until all the matrices are assigned. It should be noted that point $\mathbf{Z}(1)$ at which the permutation matrices are determined should be close to a point of degeneracy so that the error in the derivative coupling due to the nonremovable part will be small, but not so close that the energy derivatives are inaccurate. Due to the errors that arise from the neglected terms in the Taylor series and the finite number of states considered, the elements of the symmetry matrices evaluated with this procedure are only approximate. However, by comparing these estimates for the symmetry matrices to the known matrix representations of the CNPI group, it is possible to rigorously assign the symmetry matrices. This requires, for example, recognizing that an element of some $M(g)$ with a value of 0.98 should be replaced by a value of 1 , or that a value of $0.87 \ldots$ should be replaced by a value of $\sqrt{3 / 2}$.

\footnotetext{
${ }^{1}$ M. Born and R. Oppenheimer, Ann. Phys. 84, 457 (1927); H. Hettema, Quantum Chemistry: Classic Scientific Papers (World Scientific, Singapore, 2000); C. A. Mead, in Mathematical Frontiers in Computational Chemical Physics, edited by D. G. Truhlar (Springer, New York, 1988), p. 1 .
}

${ }^{2}$ M. A. Collins, Theor. Chem. Acc. 108, 313 (2002).

${ }^{3}$ L. S. Cederbaum, in Conical Intersections: Electronic Structure, Dynamics and Spectroscopy, edited by W. Domcke, D. R. Yarkony, and H. Koppel (World Scientific, Singapore, 2004).

${ }^{4}$ D. R. Yarkony, J. Chem. Phys. 112, 2111 (2000).

${ }^{5}$ C. R. Evenhuis and M. A. Collins, J. Chem. Phys. 121, 2515 (2004); C. R. Evenhuis, X. Lin, D. H. Zhang, D. R. Yarkony, and M. A. Collins, ibid. 123, 134110 (2005).

${ }^{6}$ A. J. C. Varandas, F. B. Brown, C. A. Mead, D. G. Truhlar, and N. C. Blais, J. Chem. Phys. 86, 6258 (1987).

${ }^{7}$ A. Viel, W. Eisfeld, S. Neumann, W. Domcke, and U. Manthe, J. Chem. Phys. 124, 214306 (2006).

${ }^{8}$ H. Koppel, J. Gronki, and S. Mahapatra, J. Chem. Phys. 115, 2377 (2001).

${ }^{9}$ H. Nakamura and D. G. Truhlar, J. Chem. Phys. 115, 10353 (2001); K. Ruedenberg and G. J. Atchity, ibid. 99, 3799 (1993); P. Cattaneo and M. Persico, Chem. Phys. 214, 49 (1997); T. Pacher, H. Koppel, and L. S. Cederbaum, J. Chem. Phys. 95, 6668 (1991); D. R. Yarkony, J. Phys. Chem. A 102, 8073 (1998).

${ }^{10}$ H. J. Werner and W. Meyer, J. Chem. Phys. 74, 5802 (1981).

${ }^{11}$ M. Baer, Chem. Phys. Lett. 35, 112 (1975).

${ }^{12}$ R. G. Sadygov and D. R. Yarkony, J. Chem. Phys. 109, 20 (1998).

${ }^{13}$ C. A. Mead and D. G. Truhlar, J. Chem. Phys. 77, 6090 (1982).

${ }^{14}$ B. K. Kendrick, C. A. Mead, and D. G. Truhlar, Chem. Phys. 277, 31 (2002).

${ }^{15}$ N. Matsunaga and D. R. Yarkony, Mol. Phys. 93, 79 (1998).

${ }^{16}$ R. Farwig, in Algorithms For Approximation, edited by J. C. Mason and M. G. Cox (Clarendon, Oxford, 1987), p. 194.

${ }^{17}$ J. Ischtwan and M. A. Collins, J. Chem. Phys. 100, 8080 (1994); M. J. T. Jordan, K. C. Thompson, and M. A. Collins, ibid. 102, 5647 (1995).

${ }^{18}$ K. C. Thompson, M. J. T. Jordan, and M. A. Collins, J. Chem. Phys. 108, 8302 (1998).

${ }^{19}$ R. P. A. Bettens and M. A. Collins, J. Chem. Phys. 111, 816 (1999).

${ }^{20}$ G. Herzberg, Electronic Spectra and Electronic Structure of Polyatomic Molecules, Molecular Spectra and Molecular Structure Vol. III, (Van Nostrand, Princeton, 1966).

${ }^{21}$ M. A. Collins and D. F. Parsons, J. Chem. Phys. 99, 6756 (1993).

${ }^{22}$ K. C. Thompson and M. A. Collins, J. Chem. Soc., Faraday Trans. 93, 871 (1997).

${ }^{23}$ J. C. Tully, J. Chem. Phys. 93, 1061 (1990).

${ }^{24}$ C. Zhu, S. Nangia, A. W. Jasper, and D. G. Truhlar, J. Chem. Phys. 121, 7658 (2004)

${ }^{25}$ M. S. Topaler, T. C. Allison, D. W. Schwenke, and D. G. Truhlar, J. Phys. Chem. A 102, 1666 (1998); M. D. Hack, A. W. Jasper, Y. L. Volobuev, D. W. Schwenke, and D. G. Truhlar, ibid. 103, 6309 (1999).

${ }^{26}$ W. H. Press, B. P. Flannery, S. A. Teukolsky, and W. T. Vetterling, Numerical Recipes in Fortran: The Art of Scientific Computing (Cambridge University Press, Cambridge, 1986).

${ }^{27}$ T. Vertesi, E. Bene, A. Vibok, G. J. Halasz, and M. Baer, J. Phys. Chem. A 109, 3476 (2005). 